\title{
EVOLVING STANDARDS FOR ACADEMIC PUBLISHING: A $q-r$ THEORY
}

\author{
Glenn Ellison
}

Working Paper 7805

http://www.nber.org/papers/w7805

\author{
NATIONAL BUREAU OF ECONOMIC RESEARCH \\ 1050 Massachusetts Avenue \\ Cambridge, MA 02138 \\ July 2000
}

I would like to thank the National Science Foundation (SBR-9818534), the Sloan Foundation and the Center for Advanced Studies in the Behavioral Sciences for their support. I would like to thank Robert Barro, John Cochrane, Ken Corts, Drew Fudenberg, John Geanakoplos, Robin Pemantle, Gary Saxonhouse and various seminar audiences for helpful comments and Christine Kiang and Nada Mora for outstanding research assistance. The views expressed here are the author and do not necessarily reflect those of the National Bureau of Economic Research.

(C) 2000 by Glenn Ellison. All rights reserved. Short sections of text, not to exceed two paragraphs, may be quoted without explicit permission provided that full credit, including $\mathbb{C}$ notice, is given to the source. 
Evolving Standards for Academic Publishing: A $q-r$ Theory

Glenn Ellison

NBER Working Paper No. 7805

July 2000

JEL No. A14, D70, C73

\begin{abstract}
This paper develops a model of evolving standards for academic publishing. It is motivated by the increasing tendency of academic journals to require multiple revisions of articles and by changes in the content of articles. Papers are modeled as varying along two quality dimensions: $q$ and $r$. The former represents the clarity and importance of a paper's main ideas and the latter its craftsmanship and polish. Observed trends are regarded as increases in r-quality. A static equilibrium model in which an arbitrary social norm determines how $\mathrm{q}$ and $\mathrm{r}$ are weighted is developed and used to discuss comparative statics explanations for increases in $r$. The paper then analyzes a learning model in which referees (who have a biased view of the importance of their own work) try to learn the social norm from observing how their own papers are treated and the decisions editors make on papers they referee. The model predicts that social norms will gradually but steadily evolve to increasingly emphasize r-quality.
\end{abstract}

Glenn Ellison

Department of Economics

Massachusetts Institute of Technology

50 Memorial Drive

Cambridge, MA 02142-1347

and NBER

gellison@mit.edu 


\section{Introduction}

I encourage potential readers of this paper to first put it down for two minutes and thumb through a thirty- or forty-year-old issue of an economics journal. I anticipate that this will convey much better than I can how dramatically economics papers have changed over the last few decades. Papers today are much longer. ${ }^{1}$ They have longer introductions. ${ }^{2}$ They have more sections discussing extensions of the main results. They have more references. ${ }^{3}$ The process by which papers get published has also changed dramatically. Around 1960 the Quarterly Journal of Economics reserved the "revise-and-resubmit" option for five or so special cases per year. In the early 1970's most journals' processes still let authors submit their papers, receive reports, make revisions and get a final acceptance within about nine months. Today extensive revisions are the norm and getting an acceptance takes 20 to 30 months at most top journals. ${ }^{4}$ Many other academic disciplines have had similar experiences. While the changes in academic journals are widespread and dramatic, they have received little attention. Perhaps this reflects that the changes have occurred gradually and that researchers in each field see only a small part of the overall picture.

In this paper I develop a model to organize the observed trends and help us think about potential explanations. My central premise is that we can usefully regard academic papers as differing along two quality dimensions: $q$ and $r$. I think of $q$ as reflecting a paper's main ideas and $r$ as representing other aspects of quality described by words like craftsmanship, thoroughness and polish. I think of the various changes noted above as different aspects of a single phenomenon - an increase in the $r$-quality of published papers. I first develop an equilibrium model of time allocation and quality standards and use it to discuss what

\footnotetext{
${ }^{1}$ Ariel Rubinstein's 1982 Econometrica article, "Perfect Equilibrium in a Bargaining Model," for example, is shorter than the average note in Econometrica in 2000 (if one accounts for the type sizes).

${ }^{2}$ For example, Amartya Sen's "The Impossibility of a Paretian Liberal" and two other papers in the January-February 1970 issue of the Journal of Political Economy had one paragraph introductions. No introduction in the Feburary 2000 JPE was shorter than seven paragraphs and two were longer than Sen's entire paper.

${ }^{3}$ An extreme example here is Michael Spence's 1973 QJE paper "Job Market Signaling." It contains two references. While my anectodes all relate to classic papers, old journals also contain many derivative papers making $N^{\text {th }}$ order contributions to long forgotten literatures. These also look very different from today's papers.

${ }^{4}$ The one exception is the $Q J E$ where it takes about 13 months. See Ellison (2000) for much more data on the duration of review process.
} 
might cause such an increase. I then develop a dynamic learning model in which economists struggle to reconcile the high standards to which they are being held with the mediocrity they see in journals (This occurs in part because economists have biased views of the quality of their own work.) The model predicts that social norms will evolve gradually to emphasize $r$-quality.

I begin in Section 2 with a quick look at some data on the form of papers and the length of the review and revision process in a number of disciplines.

Section 3 describes a static equilibrium model of the journal publishing process. A continuum of economists are endowed with one unit of time in which to write one paper for the one journal in the profession. Papers are described by their position along two quality dimensions, $q$ and $r$. I interpret the $q$ dimension as reflecting the clarity and importance of the main results of the paper, and the $r$ dimension as reflecting various other aspects of quality (generality, robustness checks, extensions, etc.) that are typically improved in revisions and when authors "polish" papers prior to submission. The main decision economists make is how to divide their time between the developing new ideas and working on $r$-quality. A commonly understood social norm determines how the quality dimensions are weighted.

I analyze the static model in Section 4. For a range of parameter values the model produces a reasonable outcome. Papers initially submitted to the journal can be divided into three groups. Papers with the lowest $q$ are determined to be of sufficiently low interest that no feasible revision could make them acceptable. They are not revised. A second set of papers with intermediate $q$ 's are marginal. These papers are revised to the greatest extent possible, but only some become acceptable. Finally, papers with a sufficiently high $q$ will be publishable even if they achieve a somewhat lower $r$. The authors of these papers are always able to meet the revision standard that is set for them.

I make two main observations about the static model. First, a continuum of social norms are possible. If the community agrees that q-quality is very important, then authors will spend most of their time developing main ideas. If $r$-quality is very important, then authors spend very little time on ideas and focus on revisions. Nothing in the model prevents either extreme or something in the middle from being an equilibrium. Second, the marginal papers 
that fall just short of being accepted are relatively low in q-quality and relatively high in $r$-quality relative to the set of resubmitted papers. This is a consequence of the assumption that a paper's q-quality is developed first and revisions later increase $r$.

The model makes clear that a number of equilibrium comparative statics explanations could be given for increases in $r$-quality. Such explanations, however, may not work well in practice. The observed changes in economics and other fields are large. Accounting for them requires either a large change in some exogenous variable or a high sensitivity of the outcome to an exogenous variable. My impression from the empirical work I've done is that the economics profession has not changed much over the last thirty years. (Ellison, 2000) Equilibrium explanations are also hard to reconcile with editors' comments that changes were not planned or desired. I take these difficulties as motivation for exploring evolutionary explanations - to account for observed changes such an explanation only need sto provide a good reason to think that each year papers will be a paragraph longer and require a couple weeks more to revise than the year before.

Section 5 describes a dynamic learning model. The main actors in the model are the journal's referees (who are also the authors). The referees are not self-interested. They simply try to apply the prevailing standards of the profession in weighting $q$-quality and $r$-quality and in proposing a set of improvements that would bring a paper up to the publication threshold. Referees learn the prevailing standards from two sources: observations of what revisions they are asked to make and observations of whether editors eventually decide to accept or reject papers they have refereed. Editors are not an active force in the model. They simply fill the journal's slots by accepting the fraction $\tau$ of papers with the highest average quality (weighted according to the then prevailing norm). I view this as a model of a busy editor. Making referees the driving force is also consistent with editors' comments that they abhor the trend toward fifty page papers with myriad extensions and wish that authors would just concisely explain what ideas they have.

Section 6 discusses the dynamic model with no overconfidence bias. All of the equilibria of the static model are steady-states. I note that if referees try to to hold authors to a standard that is infeasibly high, then economists will both realize that overall quality standards are lower than they had thought and conclude that $r$-quality must be relatively more 
important than they had thought. The latter inference is made because the submarginal papers that are unexpectedly accepted are relatively low in $q$ and relatively high in $r$.

Section 7 adds in the assumption that economists are biased and think their work is slightly better than it really is. ${ }^{5}$ The former steady-states of the model are destabilized as referees try to hold others to the higher standard that they perceive is being applied to their own papers. Standards can not stray far from the equilibrium set - referees would see editors accepting many papers they strongly recommended against and this would overwhelm the $\epsilon$ bias. What happens instead is that referees perpetually try to hold authors to a standard that is slighly too high. The observation of the previous section about how economists rationalize standards that are too high still applies. The result is a gradual evolution of social norms to increasingly weight $r$-quality. The slow steady form of the dynamics makes them a candidate for explaining observed trends. In the long run the process stops short of papers having no $q$-quality at all, because the dynamics change once no paper's idea is good enough to make its acceptance a sure thing.

There has been little related theoretical work on the dynamics of standards. The most notable is Sobel's (1998) analysis of a model in which a series of candidates work to obtain admission to a club. (One could think of publishing in a journal as an example.) Judges have heterogeneous tastes. The focus is on how different voting rules lead to rising, falling or fluctuating standards.

The fact that I have not to followed the current trend and given this paper an overly general title and a seven page introduction should not be taken to indicate that there are not broader lessons to be learned from it. There are all kinds of social norms, e.g. standards for politeness, standards for language and violence on television, hazing at fraternities, hours worked by young doctors and lawyers, years spent in higher education, distributions of grades, etc. Many of these norms have commonly perceived trends, but other than the

\footnotetext{
${ }^{5}$ In the psychology literature this is referred to as an overconfidence bias. An often discussed example is Svenson's (1981) finding that about 90 percent of the U.S. college students in his study estimated themselves to be safer (and more skillful) at driving than the median subject. Psychologists have reported that experts in many fields are overconfident in assessing their own ability to answer questions. Lichtenstein, Fischoff and Phillips (1982) provide a nice (but early) survey. Overconfidence is frequently mentioned in finance to motivate the existence of agency problems and to justify the actions of noise traders as in De Long et al (1990). Odean (1998) is a notable recent contribution with a detailed summary of psychology and finance papers on overconfidence. Rabin (1998) discusses a number of other psychological biases and their relevance to economics.
} 
literature on fashion cycles, e.g. Karni and Schmeidler (1990) and Pesendorfer (1995), most of the existing literature on social norms doesn't focus on change. I will not try to draw conclusions about other norms from my results. My view is that one would need to analyze each application separately and think about how the norm is learned to assess whether a drift should be expected and if so in what direction.

On a theoretical level, the paper's innovation is to note that one can produce a model that explains a long gradual trend by making a slight perturbation to a model with a continuum of equilibria. In an early presentation of this paper Robert Barro asked a penetrating question: 'so are you trying to tell us that you're going to explain a thirty-year trend by saying that we've been out of equilibrium the whole time?' My answer is "Yes!" I hope to convince readers that models of the type I introduce make such arguments possible.

\section{Some data from various disciplines}

Table 1 provides some statistical evidence on how the form of an academic article has changed. The table lists the average page length and the average number of references for articles in top journals in a number of disciplines. Economics papers are roughly twice as long as they were twenty five years ago and have about twice as many references. In almost all fields papers seem to be longer now than in 1975. The increases are more moderate in the sciences. Likewise with the exceptions of law and history, articles now tend to have more references. While economics has experienced substantial reference growth it has a long way to go to catch many social sciences.

Table 2 provides some evidence on how long it takes to review papers and on how extensively they are revised for publication in a number of fields. In almost every case it takes longer to get a paper accepted now than it did in 1975. While Econometrica and the Review of Economic Studies have the most drawn out publication processes among the listed journals, similar trends are visible in computer science, psychology, statistics, linguistics and finance, and I've been told that many rounds of revisions are also the norm at the top journals in marketing, political science, and a number of other social sciences. A slowdown is also visible in some sciences, but the time scale is completely different. 


\section{The static model}

In this section I describe a simple static model of academic publishing. The main actors in the model are a continuum of economists (of unit mass). Each economist is endowed with one unit of time and may write one paper. There is one economics journal that publishes a mass $\tau$ of papers with $0<\tau<1$. Economists preferences are lexicographic in publications and leisure time, i.e. they attempt to maximize the probability of publishing an article in the journal; holding the probability of publication fixed they prefer more leisure to less.

Papers can be fully described by two dimensions of quality, $q$ and $r$. The $q$-quality dimension is intended to reflect the inherent importance, interest, clarity etc. of the main ideas of the paper. The $r$-quality dimension is intended to reflect additional aspects of quality that authors improve when they are asked in revisions to improve and tighten a paper's exposition, to make clear relationships to other papers in the literature, to generalize theoretical results, to check the robustness of empirical results, to extend the analysis to consider related questions, etc.

Social norms for evaluating papers are assumed to be common and commonly known. Under the $(\alpha, z)$-social norm, papers are regarded as worth of publication if and only if $\alpha q+(1-\alpha) r \geq z$. The parameter $\alpha$ may reflect two different value judgements. In addition to reflecting what people think makes a paper valuable, it can also reflect what people think authors should be required to do. For example, a referee might argue that while he feels that a particular high $q$, low $r$ paper is "better" than the marginal paper in a journal, it should still be rejected because the good idea does not excuse the author's failure to make $r$ improvements required of everyone else.

The timeline of the model is illustrated below. While the model is described as a four step process with three groups of players, at the moment the authors are the only ones acting in a nonmechanical way.

\begin{tabular}{cccc}
$t=1$ & $t=2$ & $t=3$ & $t=4$ \\
\hline $\mid$ & $\mid$ & Economists & Editors \\
Economists & assess $q$ & choose & say \\
choose & report $r(q)$ & $t_{r} \in\left[0,1-t_{q}\right]$ & yes $/$ no
\end{tabular}


In the first stage of the model, authors choose the fraction $t_{q} \in[0,1]$ of their time to devote to thinking up and developing the main ideas of the paper. The result is a paper of $q$-quality $q \sim F\left(q \mid t_{q}\right)$. Assume that $F$ is continuously differentiable in $t_{q}$ and for each $t_{q} F$ has an everywhere positive density $f\left(q \mid t_{q}\right)$ on the interval $\left[0, m\left(t_{q}\right)\right]$ (with $m\left(t_{q}\right)>0$ being possibly infinite). Natural specifications will have the $q$ distribution increasing in $t_{q}$. For example, $q$ might be assumed to be uniformly distributed on $\left[0, t_{q}\right]$ or exponentially distributed with mean $t_{q}$.

In the second stage of the model authors submit their papers to the journal. The journal's referees correctly assess the quality $q$ of the paper and report that it will be acceptable for publication if and only if authors are able to revise it and achieve an $r$ quality of at least $r(q)$ as defined by $\alpha q+(1-\alpha) r(q)=z$. In practice one can think of $r(q)$ as a measure of the number of improvements refereees ask for in their reports and the difficulty of the tasks.

In the third stage authors choose the amount of time $t_{r} \in\left[0,1-t_{q}\right]$ to spend on revisions. The production of $r$-quality is again a random process. Specifically, assume that $r=h\left(t_{r}\right)+\eta$, where $\eta$ is a random variable uniformly distributed on $[0, \sigma]$ with $\sigma>0$. Assume that the production of revisions is a decreasing returns activity with $h(0)=0$, $h^{\prime} \geq 0$ and $h^{\prime \prime}<0$. To ensure that time will be allocated to both dimensions of quality I assume also that $h^{\prime}(0)=\infty$, and $h^{\prime}(1)=0$.

In the fourth stage, editors accept the fraction $\tau$ of papers for which $\alpha q+(1-\alpha) r$ is highest for publication.

An equilibrium of the model is a quadruple, $\left(\alpha, z, t_{q}^{*}, t_{r}^{*}(q)\right)$ such that $t_{q}^{*}$ and $t_{r}^{*}(q)$ are chosen to maximize the probability that $\alpha q+(1-\alpha) r \geq z$ (and are as small as possible if there are multiple choices that yield the same probability of publication) and such that the fraction of papers with $\alpha q+(1-\alpha) r \geq z$ is exactly $\tau$. I will refer to $(\alpha, z)$ as a consistent social norm if there exist choices of $t_{q}$ and $t_{r}(q)$ for which $\left(\alpha, z, t_{q}, t_{r}(q)\right)$ is an equilibrium.

\section{Analysis of the static model}

In this section I analyze the model described in the previous section. I wish to highlight two main observations. First, many different social norms are possible. Second, the marginal 
accepted papers tend to have relatively low $q$-quality and relatively high $r$-quality in the universe of published papers.

\subsection{Characterization of equilibrium}

The analysis of the equilibrium is a straightforward backward induction argument. Consider an equilibrium $\left(\alpha, z, t_{q}^{*}, t_{r}^{*}(q)\right)$. Because of the lexicographic preference for publications over leisure, at $t=3$ authors will devote all of their remaining time to improving their paper's $r$-quality unless the paper is sure to be rejected anyway or is sure to be accepted even if less time is devoted to revisions. As a result we have,

Proposition 1 In any equilibrium of the model, let $\underline{q}=\left(z-(1-\alpha)\left(h\left(1-t_{q}^{*}\right)+\sigma\right)\right) / \alpha$ and $\bar{q}=\left(z-(1-\alpha) h\left(1-t_{q}^{*}\right)\right) / \alpha$. Then, $\underline{q}<\bar{q}$ and

$$
t_{r}^{*}(q)= \begin{cases}0 & \text { if } q<\underline{q} \\ 1-t_{q}^{*} & \text { if } \underline{q}<\bar{q} \leq \bar{q} \\ h^{-1}((z-\alpha q) /(1-\alpha)) & \text { if } \bar{q} \leq q\end{cases}
$$

(For this proposition, define $h^{-1}(x) \equiv 0$ if $x<0$.)

Note that $\underline{q}$ may be less than zero and $\bar{q}$ may be greater than the upper bound of the support of $q$. Hence, it is possible that either of the extreme cases may not arise for particular parameterizations of the model.

In the first stage, the time $t_{q}$ allocated to trying to develop the main ideas for a paper will be chosen to maximize the probability of eventual publication. Write $G(z ; t)$ for the probability that $\alpha q+(1-\alpha) r$ is at most $z$ when $t_{q}=t$ and $t_{r}$ is chosen optimally as in the above proposition. Note that this probability is the same as it would be if $t_{r}$ was simply set equal to $1-t_{q}$. Hence,

$$
G(z ; t)=\int_{q=0}^{\min \left(m(t), \frac{z-(1-\alpha) h(1-t)}{\alpha}\right)} \max \left(1, \frac{z-\alpha q-(1-\alpha) h(1-t)}{\sigma}\right) f(q \mid t) d q
$$

Note that $G$ is uniformly continuous in $z$ and $t$. It is strictly increasing in $z$ whenever $G(z ; t)$ is strictly between zero and one. Write $G^{-1}(p ; t)$ for the inverse of this function for a fixed $t$. The equilibrium time allocation is easily described by 
Proposition 2 In the first stage of any equilibrium, the time allocated to developing qquality satisfies

$$
t_{q}^{*} \in \operatorname{Argmax}_{t} G^{-1}(1-\tau ; t)
$$

$\underline{\text { Proof }}$

In equilibrium, each economist's paper is accepted with probability $\tau$. Hence, $z$ must satisfy $G\left(z ; t_{q}^{*}\right)=(1-\tau)$. If $t_{q}^{*}$ does not belong to $\operatorname{Argmax}_{t} G^{-1}(1-\tau ; t)$, then any $t$ which does maximize that expression has $G^{-1}(1-\tau ; t)>G^{-1}\left(1-\tau ; t_{q}^{*}\right)=z$. Because $G$ is strictly increasing in $z$ whenever $0<G(z ; t)<1$, this implies that $G(z, t)<G\left(G^{-1}(1-\tau ; t)\right)=\tau$, which contradicts the optimality of $t_{q}^{*}$.

\section{QED.}

\subsection{Some examples}

Figure 1 illustrates the distribution of paper qualities in a "typical" equilibrium — in this case generated by assuming that $\tau=0.3, q$ is uniformly distributed on $\left[0, t_{q}\right]$, the technology for $r$ production is $h\left(t_{r}\right)=\sqrt{t_{r}}-t_{r} / 2$ with $\sigma=0.2$, and the social norm for judging papers has $\alpha=0.5$ and $z \approx 0.504$.

For these parameters, the equilibrium effort allocated to $q$ production turns out to be $t_{q}^{*} \approx 0.826$. All three possible outcomes of a submission occur. Authors of papers with $q$-quality less that $\underline{q} \approx 0.48$ realize that their papers have no chance of becoming acceptable and do not attempt to revise them. Authors of papers with $q \in(\underline{q}, \bar{q}) \approx(0.48,0.68)$ devote as much time as possible to revising (setting $t_{r}=1-t_{q}^{*} \approx 0.174$ ) and have their papers accepted with probability strictly between zero and one. Authors of papers with quality $q \in\left[\bar{q}, m\left(t_{q}\right)=t_{q}^{*}\right] \approx[0.68,0.83]$ do the minimal revision necessary to ensure that their papers will be accepted with probability one.

The figure shows the outline of the support of the equilibrium distribution of paper qualities in $(q, r)$-space. Paper qualities are distributed with a constant density within these regions. Papers in the lower left box are those for which authors set $t_{r}=0$. These papers are never accepted. The upper right region is divided into a triangle of papers that are revised then rejected and a trapezoid of papers that are accepted. The mass of papers in this upper region is, of course, $\tau$. 


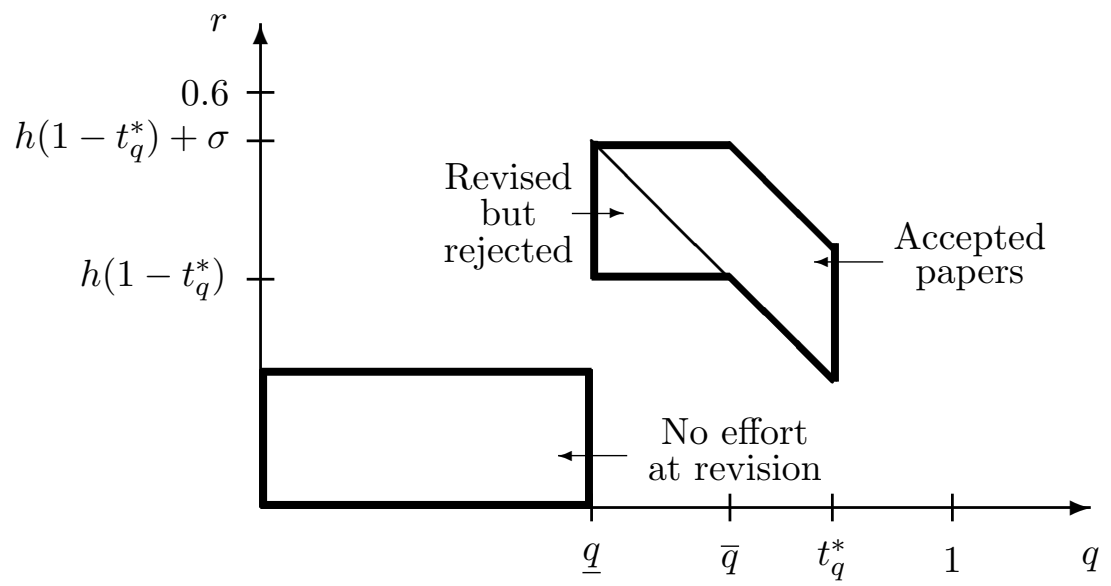

Figure 1: The equilibrium distribution of paper qualities in a "typical" equilbrium: $\tau=0.3$, $q \sim U\left[0, t_{q}\right], h(t)=\sqrt{t}-t / 2, \sigma=0.2, \alpha=0.5, z \approx 0.504$.

The form of the equilibrium seems to reflect fairly well the functioning of an economics journal. One observation I'd like to make is that the "marginal" rejected papers have relatively low $q$-quality compared to the pool of accepted papers - they all have $q \in[\underline{q}, \bar{q}]$. The marginal rejected papers are not relatively low in $r$-quality. Their authors have spent as much time as possible revising and achieved $r$-qualities that are on average superior to those of accepted papers.

While I think that the case illustrated above is the primary one of interest and subsequent arguments focus on it, the equilibrium can take other forms for different parameter values. Most notably, when $\alpha$ is sufficiently small (i.e. $q$-quality is of little importance) all authors set $t_{r}=t_{q}^{*}$ - there will always be some chance that any idea, no matter how vacuous, can be developed into a publishable paper and no paper's idea is good enough to make its eventual acceptance a sure thing. The following proposition formalizes this observation. Figure 2 graphs the equilibrium distribution of paper qualities and the acceptance and rejection regions for such a case: $q \sim U\left[0, t_{q}\right], h\left(t_{r}\right)=\sqrt{t_{r}}-t_{r} / 2, \sigma=0.2, \alpha=0.2$ and $z \approx 0.532$.

Proposition 3 Suppose that the upper bound of the $q$-distribution, $m\left(t_{q}\right)$, is finite and uniformly bounded for all $t_{q} \in[0,1]$. Then, there exists $\bar{\alpha}>0$ such that for all $\alpha \in(0, \bar{\alpha})$, $t_{r}^{*}(q)=1-t_{q}^{*}$ for all $q \in\left[0, m\left(t_{q}^{*}\right)\right]$, i.e. all papers are revised to the greatest extent possible 


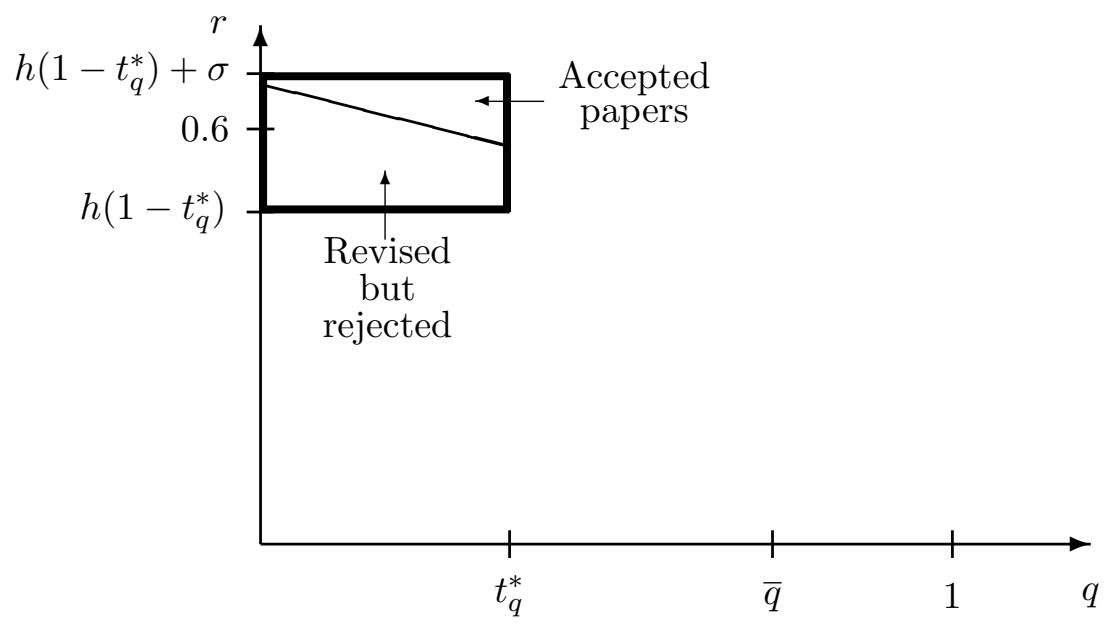

Figure 2: An example of the equilibrium quality distribution in a "low $\alpha$ " equilibrium: $\tau=0.3, q \sim U\left[0, t_{q}\right], h(t)=\sqrt{t}-t / 2, \sigma=0.2, \alpha=0.2, z \approx 0.532$.

and no paper achieves a level of q-quality sufficient to ensure that it will be accepted with probability one.

\section{$\underline{\text { Proof }}$}

To see that $\underline{q} \leq 0$ note that if a paper with $q=0$ is revised to the greatest extent possible and gets the best possible draw on $r$-quality its overall quality will be $(1-\alpha) h\left(1-t_{q}^{*}\right)+\sigma$. In equlibrium,

$$
\begin{aligned}
\operatorname{Prob}\left\{\alpha q+(1-\alpha) r \geq(1-\alpha)\left(h\left(1-t_{q}^{*}\right)+\sigma\right)\right\} & \leq \operatorname{Prob}\left\{r \geq h\left(1-t_{q}^{*}\right)+\sigma-\frac{\alpha}{1-\alpha} m\left(t_{q}^{*}\right)\right\} \\
& \leq \frac{\alpha}{1-\alpha} m\left(t_{q}^{*}\right) / \sigma \rightarrow 0 \text { as } \alpha \rightarrow 0
\end{aligned}
$$

For $\alpha$ sufficiently small the expression is less than $\tau$, and hence there is a positive probability of the paper with $q=0$ being acceptable.

Similarly, to see that $\bar{q}>m\left(t_{q}^{*}\right)$ note that if a paper with $q$-quality $m\left(t_{q}^{*}\right)$ is revised to the greatest extent possible but gets the worst possible draw on $r$-quality its overall quality level is $\alpha m\left(t_{q}^{*}\right)+(1-\alpha) h\left(1-t_{q}^{*}\right)$ and

$$
\begin{aligned}
\operatorname{Prob}\left\{\alpha q+(1-\alpha) r \geq \alpha m\left(t_{q}^{*}\right)+(1-\alpha) h\left(1-t_{q}^{*}\right)\right\} & \geq \operatorname{Prob}\left\{r \geq h\left(1-t_{q}^{*}\right)+\frac{\alpha}{1-\alpha} m\left(t_{q}^{*}\right)\right\} \\
& \geq 1-\frac{\alpha m\left(t_{q}^{*}\right)}{\sigma(1-\alpha)} \rightarrow 1 \text { as } \alpha \rightarrow 0
\end{aligned}
$$


Hence, for any $\tau<1$ the probability that a paper with $q$-quality $m\left(t_{q}^{*}\right)$ fails to be among the best $\tau$ is strictly positive if $\alpha$ is sufficiently small.

\section{QED.}

Some of the results I'll give later will depend on the form of the equilibrium. To simplify the statements of these results I will give the forms pictured in the figures (and a couple of other forms) names.

Definition 1 I will say that an equilibrium is "typical" or has the typical form if $0<\underline{q}<$ $\bar{q}<m\left(t_{q}^{*}\right)$ and $r\left(m\left(t_{q}^{*}\right)\right)>0$. An equilibrium has the "low $\alpha$ " form if $\underline{q}<0<m\left(t_{q}^{*}\right)<\bar{q}$.

It has the "somewhat low $\alpha$ " form if $0<\underline{q}<m\left(t_{q}^{*}\right)<\bar{q}$. It is of the "high $\alpha$ " form if $0<\underline{q}<\bar{q}<m\left(t_{q}^{*}\right)$ and $r\left(m\left(t_{q}^{*}\right)\right)<0$.

The somewhat low $\alpha$ form is similar to the low $\alpha$ form, with the only difference being that in the former authors of the lowest $q$ papers do not revise their papers. The high $\alpha$ form is similar to the typical form, but the highest $q$ papers are so good that $r(q)$ is negative, i.e. referees tell the authors that even if they revised the paper to make it worse it would still be publishable. The authors of these papers obviously exert no effort on revisions and have their papers accepted with quality to spare. In the model with $q \sim U\left[0, t_{q}\right], h(t)=\sqrt{t}-t / 2$, $\sigma=0.2$ and $\tau=0.3$ the equilibrium has the low $\alpha$ form for $\alpha \in(0,0.2285)$, the somewhat low $\alpha$ form for $\alpha \in(0.2286,0.3363)$, the typical form for $\alpha \in(0.3364,0.5869)$ and the high $\alpha$ form for $\alpha \in(0.5870,1)$. In other specifications for the model the equilibrium can take on other forms. For example, if the distribution of $q$ is unbounded (or $\tau$ is large) we will simultaneously see papers of the lowest $q$-quality being resubmitted and papers of the highest q-quality being accepted with no revisions.

\subsection{The multiplicity of consistent social norms}

In the model described above not all social norms are consistent. If there is only room in the journal for a small fraction of papers, then the quality threshold $z$ must be high. This, however, is really the only constraint. Nothing in the model restricts the weight the community places on $q$-quality versus $r$-quality. There are a continuum of consistent social norms with any $\alpha$ being possible. The following proposition gives a formal statement to this 
effect, and Figure 3 graphs the set of consistent social norms for the model with $q \sim U\left[0, t_{q}\right]$, $h(t)=\sqrt{t}-t / 2, \sigma=0.2$ and $\tau=0.3$.

Proposition 4 In the model described above, for any $\alpha \in[0,1]$ there exists an unique $z^{*}(\alpha)$ such that $\left(\alpha, z^{*}(\alpha)\right)$ is a consistent social norm.

\section{Proof}

For any fixed $\alpha$, let $G(z ; t)$ be the CDF of $\alpha q+(1-\alpha) r$ as above. Let $H(z)=\inf _{t} G(z ; t)$. Because $G$ is uniformly continuous, $t$ is chosen from a compact set and $\lim _{z \rightarrow \infty} G(z ; t)=1$ for all $t, H$ is continuous with $H(0)=0$ and $\lim _{z \rightarrow \infty} H(z)=1$. Hence there is a solution $z^{*}(\alpha)$ to $H(z)=1-\tau .\left(\alpha, z^{*}(\alpha)\right)$ is a consistent social norm.

It is not possible for both $(\alpha, z)$ and $\left(\alpha, z^{\prime}\right)$ to be consistent social norms with $z<z^{\prime}$. In that case, an agent setting $t_{q}$ equal to the the equilibrium choice under the $\left(\alpha, z^{\prime}\right)$ norm would surpass the $z$ threshhold with probability greater than $\tau$.

\section{QED.}

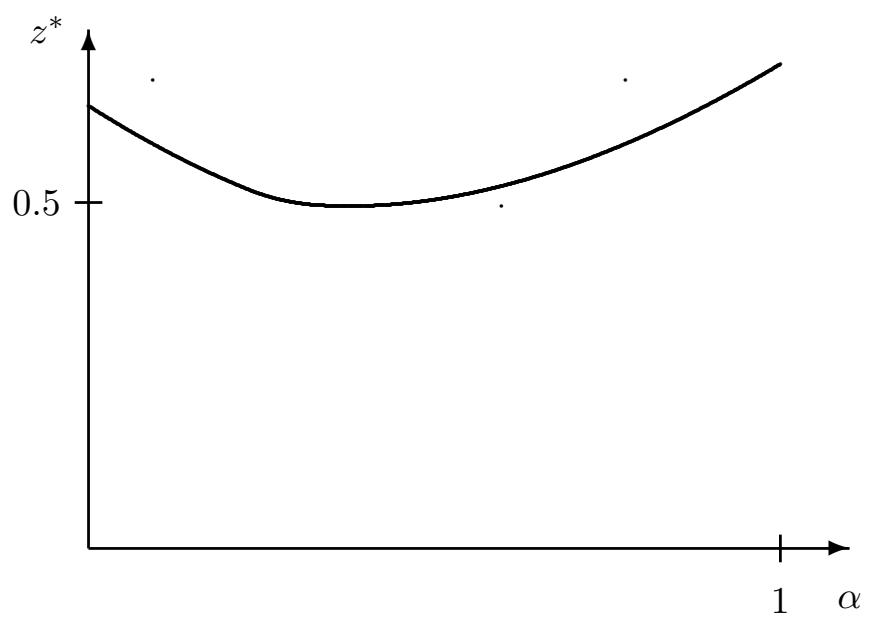

Figure 3: Consistent social norms $(\alpha, z)$ in the model with $q \sim U\left[0, t_{q}\right], h(t)=\sqrt{t}-t / 2$, $\tau=0.3$, and $\sigma=0.2$.

\subsection{Explanations for observed increases in $r$-quality}

My view of the trends in economics papers mentioned in the introduction is that they can all be thought of as aspects of an increase in the $r$-quality of published papers. The model makes clear that a number of different explanations for such a change are possible. 
The explanation I focus on in the latter half of this paper is that the $r$-quality of published papers will increase if $\alpha$ decreases, i.e. if social norms shift to place relatively less emphasis on $q$-quality and relatively more emphasis on $r$-quality. This raises the $r$-quality of published papers for two reasons: economists react by allocating more of their time to producing $r$-quality; and when choosing from the pool of resubmitted papers the journal places more emphasis on $r$-quality.

Other plausible explanations do not require a change in social norms for trading off $q$ and $r$. One is that the technologies for producing $q$ and $r$ may have shifted over time. For example, one could argue that economists have begun to exhaust the set of all possible ideas and model this as a change in $F\left(q \mid t_{q}\right)$ that reduces the marginal benefit of $t_{q}$. Similarly, one could argue that advances in computer technology or accumulated knowledge allow authors to produce more $r$-quality per unit time. A second potential explanation is that publishing in the top journals may have become more competitive. We might think of the growth of the profession and other factors as equivalent to a decrease in $\tau$, and this might raise quality standards in both dimensions. ${ }^{6}$

The latter explanation is not straightforward, so I'll discuss it further. Intuitively, reducing $\tau$ has two effects. First, it allows the journal to be more selective when choosing from the pool of resubmitted papers. Second, it affects authors' time allocation decisions. If authors react to increased competition by gambling on bold projects that require a lot of $t_{q}$, the second effect could offset the first and make the overall effect of competition on $r$-quality ambiguous.

Formally, I should note first that nothing in the assumptions so far guarantees that $G^{-1}\left(1-\tau ; t_{q}\right)$ (which plays the role of the economists' objective function when $t_{q}$ is selected) is quasiconcave. If, for example, $q \sim U\left[0, k t_{q}^{n}\right]$ with $n>1$, then the production of $q$-quality would involve increasing returns. In some cases, both both 0 and some higher value of $t_{q}$ will be local optima and $t_{q}^{*}$ will change discontinuously in the parameters. To provide as simple a discussion as I can of the potential effects of $\tau$ on $r$-quality, I will avoid such complications by just assuming that $F(q ; t)$ is such that $t_{q}^{*}$ is the unique solution to $\frac{\partial G}{\partial t}\left(z^{*}(\alpha, \tau) ; t\right)=0$

\footnotetext{
${ }^{6}$ Ellison (2000) notes that by many measures the growth in the profession over the last 30 years has been
} moderate, but that decreases in the number of articles published by some top journals and an increase in the relative status of the top journals may have also contributed to a more substantial increase in competition. 
among values of $t$ for which $G\left(z^{*}(\alpha, \tau) ; t\right)<1$ and that the probably of achieving the standard is strictly concave at the optimum, i.e. $\frac{\partial^{2} G}{\partial t^{2}}\left(z^{*}(\alpha, \tau) ; t_{q}^{*}\right)>0$.

Recall that $t_{q}^{*}(\tau)$ solves

$$
\frac{\partial G}{\partial t}\left(z^{*}(\tau) ; t_{q}^{*}(\tau)\right)=0
$$

Differentiating with respect to $\tau$ gives

$$
\frac{\partial^{2} G}{\partial z \partial t}\left(z^{*}(\tau) ; t_{q}^{*}(\tau)\right) \frac{d z^{*}}{d \tau}+\frac{\partial^{2} G}{\partial t^{2}} \frac{d t_{q}^{*}}{d \tau}=0
$$

$z^{*}$ is always decreasing in $\tau$ and we have assumed that $\frac{\partial^{2} G}{\partial t^{2}}$ is positive. Hence, $t_{q}^{*}$ will be decreasing in $\tau$ if $\frac{\partial^{2} G}{\partial z \partial t}\left(z^{*}(\tau) ; t_{q}^{*}(\tau)\right)<0$. To think about the sign of this derviative note that $\frac{\partial G}{\partial z}\left(z ; t_{q}\right)$ is the density of the overall quality distribution. If the $q$ distribution becomes more spread out when authors spend more time thinking of new ideas and the production of $r$-quality is a more deterministic process in which increases in $t_{r}$ only shift the mean $r$-quality (as we've assumed), then the natural outcome is for the density to be decreasing in $t_{q}$, in which case the $\frac{\partial}{\partial t} \frac{\partial G}{\partial z}$ is negative. In this case, $t_{q}^{*}$ will increase as $\tau$ decreases, and the overall effect of $\tau$ on $r$-quality may be ambiguous.

For a more direct intuition note that $t_{q}^{*}$ reflects authors setting $\alpha$ times the effect of $t_{q}$ on $q$-quality equal to $(1-\alpha)$ times the effect of $t_{r}$ on $r$-quality conditional on their luck being such that their paper is marginal for the journal. When the journal is highly nonselective, this is conditioning on getting a very bad draw from the $q$ distribution. When the journal is extremely selective, the conditioning is on getting nearly as a good a draw as possible on $q$. With a functional form like $F\left(q \mid t_{q}\right) \sim U\left[0, t_{q}\right]$, the higher percentiles of the $q$ distribution are more sensitive to $t_{q}$ investements. Hence the return to $t_{q}$ is greater when one conditions on the paper being marginal for a more selective journal.

Figure 4 illustrates the effects of changes in $\tau$ on time allocation and quality in a model with $q \sim U\left[0, t_{q}\right], h(t)=\sqrt{t}-t / 2, \sigma=0.2$, and $\alpha=0.5$ The left panel shows that $t_{q}^{*}$ is monotonically decreasing, i.e. $t_{q}^{*}$ increases as the journal becomes more competitive. ${ }^{7}$ The right panel shows the effect of $\tau$ on the mean $q$ - and $r$-quality of the published papers. The

\footnotetext{
${ }^{7}$ The shape of the figure when $\tau$ is near zero is sensitive to the distributional assumptions. With the uniform specification the maximum possible return to $t_{q}$ is bounded and $t_{q}^{*}$ is bounded away from one as $\tau$ approaches zero. If $q$ had been specified as exponentially distributed with parameter $t_{q}$, the effect of $t_{q}$ on the $1-\tau^{\text {th }}$ percentile of the $q$ distribution would grow without bound as $\tau$ approaches zero and the time spent on revisions would decrease all the way to zero in the limit.
} 
relationship beween $\tau$ and $r$-quality is nonmonotonic. At some points the effect of $\tau$ on $t_{q}^{*}$ dominates, while at others the effect of journal selectivity dominates.

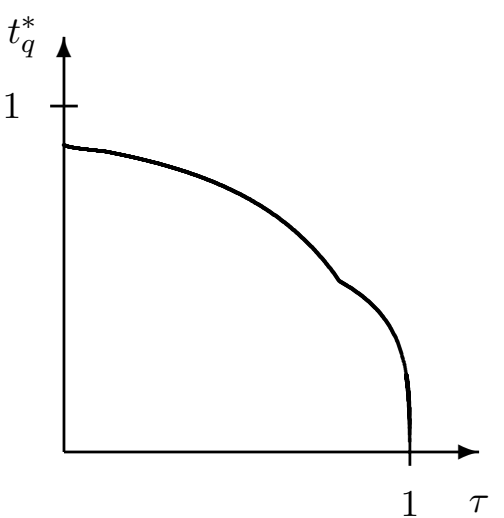

Time allocation

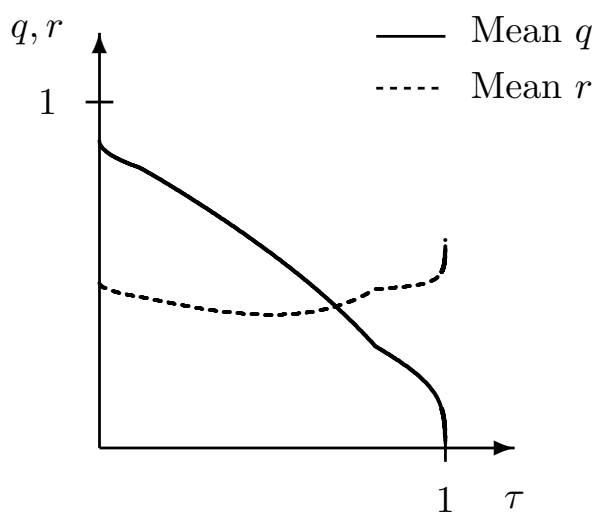

Mean quality of published papers

Figure 4: Effect of journal selectivity on time allocation and observed paper quality

\section{A dynamic model of evolving norms}

The static model above has a continuum of equilibria corresponding to different social norms. In this section I describe a dynamic model of the evolution of norms. The model involves a population of author-referees who are trying to learn and apply the profession's standards. In contrast to what one might think when one hears the term "learning model", I will not model agents as arriving with different beliefs and examine whether there is convergence to a common belief. Instead, the model will be constructed so that agents will have common beliefs at every point in time and I will focus on whether agents' attempts to learn the prevailing norm lead to a shift in norms. ${ }^{8}$

The model involves a discrete set of time periods $t=0,1,2, \ldots$ At the start of period $t$ all economists believe that that the social norm is $\left(\alpha_{t}, z_{t}\right)$. They then write a paper and try to publish it as in the static game of Section 2 and serve as a referee. The data they receive via referee reports and editorial decisions will suggest to them that the social norm

\footnotetext{
${ }^{8}$ This focus is not intended to suggest that whether a community will reach a common norm and the effects of belief or preference heterogeneity on the evolution of social norms are not interesting. Instead, as in Ellison and Fudenberg (2000), the common beliefs learning model is motivated solely by the desire for a tractable model that highlights an important effect.
} 
is in fact, $\left(\hat{\alpha}_{t}, \hat{z}_{t}\right)$. This leads them to alter their beliefs according to

$$
\left(\begin{array}{c}
\alpha_{t+1}-\alpha_{t} \\
z_{t+1}-z_{t}
\end{array}\right)=k\left(\begin{array}{c}
\hat{\alpha}_{t}-\alpha_{t} \\
\hat{z}_{t}-z_{t}
\end{array}\right)
$$

for some constant $k>0$. To complete the specification I must describe what data economists gather and how they draw the inferences $\left(\hat{\alpha}_{t}, \hat{z}_{t}\right)$ from the data.

What data do economists get? I assume that economists get two types of data points. First, when an author submits a paper of quality at least $\underline{q}$ I assume that the referee reports he or she receives give him or her a data point of the form $(q, r(q)) .{ }^{9}$ These datapoints should all lie on the line $\alpha_{t} q+\left(1-\alpha_{t}\right) r(q)=z_{t}$. I will allow, however, for the possibility that economists are subject to an overconfidence bias when judging the quality of their own work. In particular, I'll assume that they overestimate the $r$-quality of their initial submission and this leads them to believe that they have been required to achieve an $r$ quality that is $\epsilon$ higher than what they have actually been required to achieve. ${ }^{10}$ In this case, the $(q, r(q))$ datapoints actually lie on the line $\alpha_{t} q+\left(1-\alpha_{t}\right) r(q)=z_{t}+\left(1-\alpha_{t}\right) \epsilon$.

Second, whenever an economist referees a paper that is of sufficiently high quality to be resubmitted he or she gets a data point of the form ( $q, r$, Accept/Reject). ${ }^{1112}$ Economists expect to see all papers lying above the line $\alpha_{t} q+\left(1-\alpha_{t}\right) r=z_{t}$ being accepted and all papers lying below this line being rejected.

If economists each saw a finite number of data points, then their analyses would lead to a divergence in the second period beliefs even if they entered the model with common beliefs. To keep the model tractable I avoid this by invoking word-of-mouth communication.

\footnotetext{
${ }^{9}$ Note that in a slight departure from the static model I've assumed that journals do not provide the author with a list of revisions sufficient to make the paper publishable if the paper's q-quality is so low as to make it inconceivable that a revision will be publishable. I believe that this is a good description of journal practices. I did not try to incorporate such behavior by referees in the static model, however, because it seemed a needless complication and because it creates a possibility for another type of equilibrium multiplicity that I did not feel was important: authors won't save time for revisions if referees won't ask for large revisions because they don't think they're feasible.

${ }^{10}$ Given that the acceptance fronteir is downward sloping in $q-r$ space this assumption is almost equivalent to assuming that authors believe their papers to be of slightly higher $q$-quality than they actually are, or to assuming that have biased views of both the $q$ - and $r$-quality of their work. I've chosen the formulation above because it makes some results a little cleaner (especially those about small $\alpha$ behavior.)

${ }^{11}$ Another important data source for real world economists is reading journals. Given the word-of-mouth assumption below, this would just provide redundant observations on all of the acceptances. For this reason, nothing would be changed if I included this data source in the model.

${ }^{12} \mathrm{An}$ ambiguity in the model is what happens if the assumed standards is so excessively high that fewer than $\tau$ papers are resubmitted. In the simulations in the next section I assume that in this case editors accept some papers that are not resubmitted and that these acceptances are observed by the referees.
} 
I assume that each economist talks to every other economist in each period and thereby sees all of the data points that were generated in that period. While this makes each author's own experiences a measure zero subset of his or her dataset, I do not want to lose the possibility of inferences being affected by the authors' misperceptions of the quality of their own work. I thus assume that the $(q, r(q))$ observations economists receive by hearing others talk about the referee reports they received are contaminated by the author's bias (and that the listeners do not realize this).

What do economists do with the data they obtain each period? Fitting the $(\alpha, z)$ model involves estimating the slope and intercept of a line that fits the $(q, r(q))$ data and divides the acceptance and rejection regions. Typically, no line will do both jobs perfectly. I assume that this does not cause economists to lose faith in their model of the world and that they go ahead and try to fit the data as well as possible with the $(\alpha, z)$ model. ${ }^{13}$

Formally, I assume that economists' period $t$ analyses take the form

$$
\left(\hat{\alpha}_{t}, \hat{z}_{t}\right)=\arg \min _{\alpha, z} L\left(\alpha, z ; \mu_{1}, \mu_{2}\right)
$$

where $L(\alpha, z)$ is a loss function that describes how poorly the the data (a measure $\mu_{1}$ describing the $(q, r(q))$ points and a measure $\mu_{2}$ describing the $(q, r$, Accept/Reject) points) fit the hypothesis that all referees and the journal editor are applying the $(\alpha, z)$ social norm. Specifically, I assume that

$$
L\left(\alpha, z ; \mu_{1}, \mu_{2}\right)=L_{1}\left(\alpha, z ; \mu_{1}\right)+L_{2}\left(\alpha, z ; \mu_{2}\right),
$$

where

$$
L_{1}\left(\alpha, z ; \mu_{1}\right)=\int\left(r(q)-\frac{z-\alpha q}{1-\alpha}\right)^{2} d \mu_{1}(q)
$$

is a standard mean squared deviation measure of the distance (in the $r$-dimension) between the $(q, r(q))$ data points and the line $\alpha q+(1-\alpha) r=z$ and

$$
L_{2}\left(\alpha, z ; \mu_{2}\right)=\int_{R_{U A}}\left(\frac{z-\alpha q}{1-\alpha}-r\right) d \mu_{2}(q, r)+\int_{R_{U R}}\left(r-\frac{z-\alpha q}{1-\alpha}\right) d \mu_{2}(q, r)
$$

\footnotetext{
${ }^{13}$ Formally, economists are estimating a misspecified model. A justification for not worrying about economists noticing the misspecification is that in a more realistic model economists would only receive a finite number of datapoints and there would be a random component to each observation, so the form of the misspecification would not be so apparent. The idea that economists struggle to reconcile hard-toreconcile observations while maintaining biased self images does not seem unrealistic to me.
} 
where $R_{U A}$ is the set of $(q, r)$ values for which papers were "unexpectedly accepted" despite failing to meet the $(\alpha, z)$ standard and $R_{U R}$ is the set of "unexpectedly rejected" papers that met the $(\alpha, z)$ standard but were rejected. $L_{2}$ can be thought of as the product of the fraction of accept/reject decisions that are inconsistent with the $(\alpha, z)$ model and the average degree of error (in the $r$-dimension) that appears to be embodied in the "unexpected" decisions.

Obviously other loss functions would be reasonable. The most natural would probably be the negative of the log likelihood of the data under a hypothesis involving referees and the editor trying to apply the $(\alpha, z)$ norm but making idiosyncratic errors in judging the quality of each paper. Analyzing such a specification would require examinining integrals of CDF's and PDF's, however, and I felt that the specification above was the best compromise in terms of reflecting a similar goodness-of-fit notion and being tractable.

\section{Analysis of the dynamic model with no overconfidence bias $(\epsilon=0)$}

In this section I discuss the behavior of the dynamic model when economists do not have an inflated view of the quality of their own work. The main observations are that consistent social norms are steady states of the model and that when referees are too demanding economists infer both that their standards were too high and that q-quality must be relatively less important than they had thought.

\subsection{Steady states}

When there is no overconfidence bias it is easy to see that any consistent social norm of the static model is a steady state of the dynamic learning model.

Proposition 5 Suppose $\epsilon=0$ in the dynamic model and $\left(\alpha_{0}, z_{0}\right)$ is a consistent social norm. Then, $\left(\alpha_{t}, z_{t}\right)=\left(\alpha_{0}, z_{0}\right)$ for all $t$.

$\underline{\text { Proof }}$

All points $(q, r(q))$ in the data obtained from referees' reports lie exactly on the line $\alpha_{0} q+\left(1-\alpha_{0}\right) r(q)=z_{0}$. The editor's decisions are also consistent with imposing the $\left(\alpha_{0}, z_{0}\right)$ 
standard, i.e. all rejected papers have $\alpha_{0} q+\left(1-\alpha_{0}\right) r-z_{0} \leq 0$ and all accepted papers have $\alpha_{0} q+\left(1-\alpha_{0}\right) r-z_{0} \geq 0$. Hence, both $L_{1}$ and $L_{2}$ are zero for $(\alpha, z)=\left(\alpha_{0}, z_{0}\right)$. Because the $q$-distribution is nonatomic on a continuous support, $L_{1}$ is strictly positive (and $L_{2}$ is always nonnegative) for any other $(\alpha, z)$. The unique minimum of the loss function is thus $\left(\hat{\alpha}_{0}, \hat{z}_{0}\right)=\left(\alpha_{0}, z_{0}\right)$.

\section{QED.}

\subsection{Disequilibrium dynamics}

In this section I discuss the disequlibrium behavior of the dynamic model. The results in this section (and in the remainder of the paper) will concern the uniform technology for $q$-production, $q \sim U\left[0, t_{q}\right]$. The whole of what I want to say in this section can be summarized concisely by saying that the dynamic evolution of economists' beliefs about the social norm $(\alpha, z)$ outside of equilibrium follows the pattern illustrated in Figure 5. The figure was constructed by solving the model numerically for various initial beliefs under the assumption that $q \sim U\left[0, t_{q}\right], \tau=0.3, \sigma=0.2$ and $h(t)=\sqrt{t}-t / 2$. The solid line in the figure is the locus of consistent social norms $\left(\alpha, z^{*}(\alpha)\right)$. The vectors in the figure are proportional to the change in beliefs $\left(\alpha_{t+1}-\alpha_{t}, z_{t+1}-z_{t}\right)$ that occurs for various initial beliefs. ${ }^{14}$ To help organize the dynamics I have also placed a vertical dashed line in the figure at $\alpha=0.3363$. This is where the equilibrium shifts from the somewhat low $\alpha$ form to the typical form.

The locus of consistent social norms and the dashed line divide $(\alpha, z)$ space into four regions. In three of the four regions the learing process is mostly just a straightforward adjustment of the overall quality threshold: when referees try to impose a standard that would not allow the editor to fill the journal they infer from the unexpected acceptances that they must reduce $z$; when referees are too soft and the editor has to turn down some papers they recommend they learn to choose a higher $z .^{15}$

\footnotetext{
${ }^{14}$ The speed with which beliefs change in the model is determined by an arbitrary scaling parameter $k$. The figure uses $k=0.6$. Note that unlike all other graphs in this paper the $x$ and $y$ axes in this figure do not have the same scale. The $y$-axis has been magnified by a factor of 1.5 . These choices reflect an attempt to maximize the visibility of the directions and minimize clutter.

${ }^{15}$ The dynamics in the $z<z^{*}(\alpha)$ and $\alpha$ large region fit this description only if $\alpha$ is not too large. I discuss what happens in the "high $\alpha$ " case at the end of this section.
} 


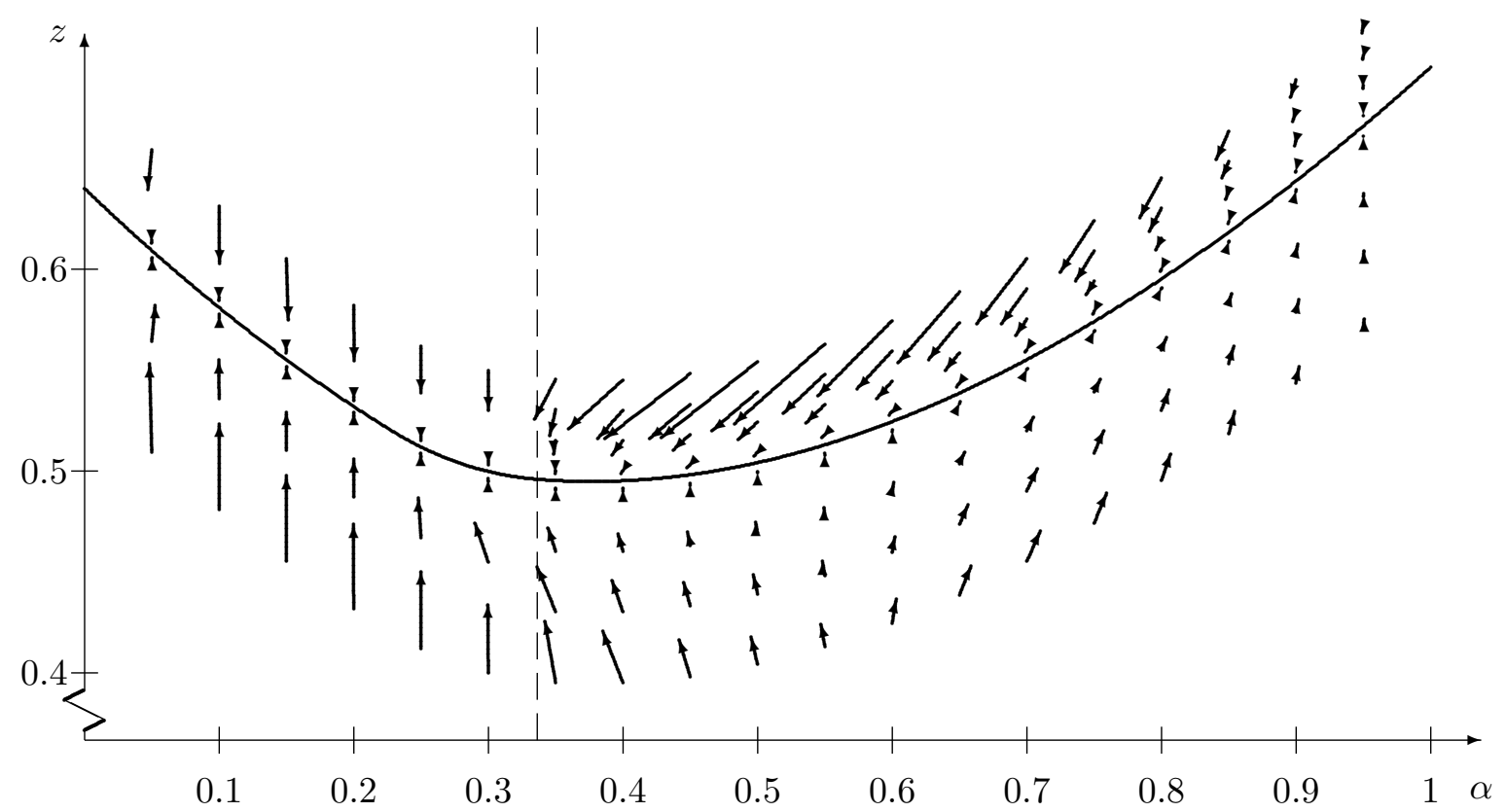

Figure 5: The disequilibrium evolution of social norms when $\epsilon=0$.

What is most important to my main argument is what happens in the fourth region - the upper right part of Figure 5. Suppose that the $\left(\alpha_{t}, z_{t}\right)$ standard is unreasonably high, i.e. $z>z^{*}(\alpha)$. Suppose also that the distribution of resubmitted papers has the "typical" form. Economists will then (correctly) perceive that referees are asking them to meet a very high standard and that some papers that they thought were submarginal are being accepted. The conflict in this data leads economists to do two things: to believe that quality standards are less demanding than they had thought; and to believe that $r$-quality is relatively more important than than they had thought.

To illustrate why economists make this inference, Figure 6 contains an enlarged view of the data economists get in one such case. ${ }^{16}$ The bold line represents the $(q, r(q))$ datapoints they get from referee reports. The outlined area is the support of the (uniform) quality distribution. All papers above and to the right of the bold line are accepted. The journal editor also accepts papers in the shaded region (to the surprise of the referees). Lower quality papers are rejected. The lines below the $q$-axis and to the left of the $r$-axis

\footnotetext{
${ }^{16}$ The figure graphs the quality distribution and the best fit $(\hat{\alpha}, \hat{z})$ when initial beliefs are that $\alpha=0.5$ and $z=0.53417$ with $q \sim U\left[0, t_{q}\right], h(t)=\sqrt{t}-t / 2, \sigma=0.2$ and $\tau=0.3$.
} 
illustrate the support of the $q$ and $r$ distributions among resubmitted papers. I have used bold lines to show the support of the quality distribution for the unexpectedly accepted papers. These papers are at the low end of the $q$ distribution and at the high end of the $r$ distribution. Economists' rationalize these acceptances in part by concluding that $r$ must be more important than they had thought. The dashed line graphs the social norm $(\hat{\alpha}, \hat{z})$ that best fits the data. The flatter line accounts for many unexpected acceptances and gives a good fit to the high $q$ part of the $(q, r(q))$ data.

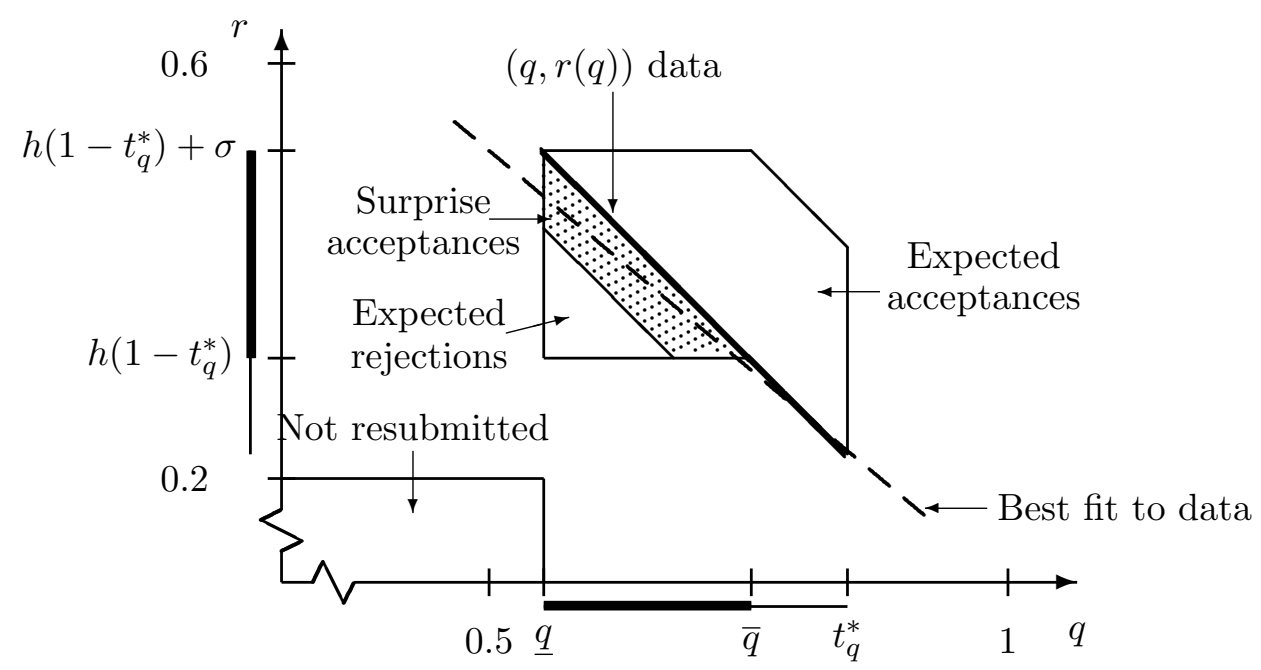

Figure 6: Economists' inferences when standards are too high: the "typical" case

Figure 7 contains a similar diagram illustrating economists inferences when referees' beliefs $\left(\alpha_{t}, z_{t}\right)$ are unreasonably tough and $\alpha_{t}$ is sufficently low so that the distribution of resubmitted papers has the "low $\alpha$ " form - all papers are resubmitted and all authors devote the same maximum effort to their revisions. Here, the "unexpectedly accepted" papers are uniformly distributed in the $q$ dimension, and the dotted line illustrates that the best fit is obtained by slightly lowering $z$ while leaving the slope of the line unchanged.

Even with the simple loss function I've chosen, getting analytic expressions for the optimal inference given an inconsistent $\left(\alpha_{t}, z_{t}\right)$ is difficult. Proposition 6 is a characterization of the dynamics that brings out the main observations I've mentioned above. The proposition characterizes the dynamics for initial beliefs that are close to being consistent, i.e. for $z_{t}$ close to $z^{*}\left(\alpha_{t}\right)$. Parts (a) and (b) note that in the low $\alpha$ and somewhat low 


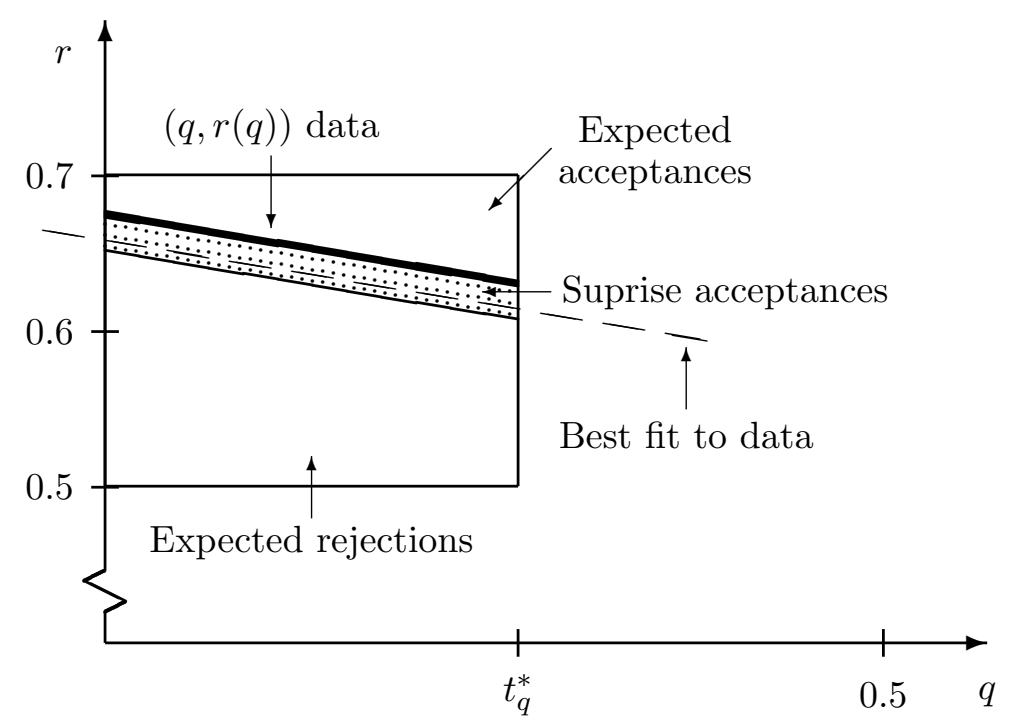

Figure 7: Inferences when standards are too high: the "low $\alpha$ " case.

$\alpha$ cases economists do not adjust their estimate of $\alpha$ (at least approximately) and adjust their estimate of $z$ toward $z^{*}\left(\alpha_{t}\right)$. Part (c) notes that when referees' beliefs correspond to a standard that is too low the dynamics are similar in the "typical" case: the dynamics are approximately vertical when the social norm is approximately consistent. Part (d) relates to my main observation. It notes that when $z_{t}$ is slightly larger than $z^{*}\left(\alpha_{t}\right)$ the dynamics involve both a reduction in $\alpha$ and a reduction in the overall quality standard (after accounting for the change that is induced mechanically by the change in $\alpha$ ). The shifts in the two parameters are comparable in magnitude. The proof of the proposition is contained in Appendix A.

Proposition 6 Consider the dynamic model described above with $F\left(q \mid t_{q}\right) \sim U\left[0, t_{q}\right]$. Let $t_{q}(\alpha, z)$ be economists' optimal time allocation when they believe that the social norm is $(\alpha, z)$. Write $b(z) \approx a\left(z-z^{*}(\alpha)\right)$ as shorthand for $\lim _{z \rightarrow z^{*}(\alpha)} \frac{b(z)}{z-z^{*}(\alpha)}=a$.

(a) Suppose that for a given $\alpha_{t} \in(0,1)$ the unique equilibrium for the social norm $\left(\alpha_{t}, z^{*}\left(\alpha_{t}\right)\right)$ has the low $\alpha$ form. Then there exists a constant $a>0$ such that for $z_{t}$ in a neighborhood of $z^{*}\left(\alpha_{t}\right)$ the dynamics have

$$
\begin{aligned}
\alpha_{t+1}-\alpha_{t} & =0 \\
z_{t+1}-z_{t} & =a\left(z^{*}\left(\alpha_{t}\right)-z_{t}\right)
\end{aligned}
$$


(b) Suppose that for a given $\alpha_{t} \in(0,1)$ the unique equilibrium for the social norm $\left(\alpha_{t}, z^{*}\left(\alpha_{t}\right)\right)$ has the somewhat low $\alpha$ form and that $\frac{\partial^{2} G}{\partial t^{2}}$ is strictly positive at $t=t_{q}\left(\alpha_{t}, z^{*}\left(\alpha_{t}\right)\right)$. Then there exists a constant $a>0$ such that for $z_{t}$ close to $z^{*}\left(\alpha_{t}\right)$ the dynamics have

$$
\begin{aligned}
\alpha_{t+1}-\alpha_{t} & \approx 0 \\
z_{t+1}-z_{t} & \approx a\left(z^{*}\left(\alpha_{t}\right)-z_{t}\right)
\end{aligned}
$$

For $z_{t}$ slightly larger than $z^{*}\left(\alpha_{t}\right)$ the dynamics have $\alpha_{t+1}-\alpha_{t}=0$.

(c) Suppose that for a given $\alpha_{t} \in(0,1)$ the unique equilibrium for the social norm $\left(\alpha_{t}, z^{*}\left(\alpha_{t}\right)\right)$ has the "typical" form and $z_{t}<z^{*}\left(\alpha_{t}\right)$. Then there exists a constant a $>0$ such that for $z_{t}$ close to $z^{*}\left(\alpha_{t}\right)$ the dynamics have

$$
\begin{aligned}
\alpha_{t+1}-\alpha_{t} & \approx 0 \\
z_{t+1}-z_{t} & \approx a\left(z^{*}\left(\alpha_{t}\right)-z_{t}\right)
\end{aligned}
$$

(d) Suppose that for a given $\alpha_{t} \in(0,1)$ the unique equilibrium for the social norm $\left(\alpha_{t}, z^{*}\left(\alpha_{t}\right)\right)$ has the "typical" form and $z_{t}>z^{*}\left(\alpha_{t}\right)$. Let $M_{q}=\left(\underline{q}\left(\alpha_{t}, z_{t}\right)+t_{q}\left(\alpha_{t}, z_{t}\right)\right) / 2$ and $M_{r}=$ $\frac{z_{t}-\alpha_{t} M_{q}}{1-\alpha_{t}}$. Then there exist constants $a_{1}>0$ and $a_{2}>0$ such that for $z_{t}$ close to $z^{*}\left(\alpha_{t}\right)$ the dynamics have

$$
\begin{aligned}
\alpha_{t+1}-\alpha_{t} & \approx-a_{1}\left(z_{t}-z^{*}\left(\alpha_{t}\right)\right) \\
z_{t+1}-z_{t} & \approx a_{2}\left(z^{*}\left(\alpha_{t}\right)-z_{t}\right)+\left(\alpha_{t+1}-\alpha_{t}\right)\left(M_{q}-M_{r}\right)
\end{aligned}
$$

The one notable feature of the Figure 5 that I have left out of the discussion so far is what happens when $z<z^{*}(\alpha)$ and $\alpha$ is sufficiently high so that the equilibrium has the high $\alpha$ form It is apparent from the figure that in this case economists conclude that $q$ is relatively more important than they had thought. The argument is similar to the argument for why economists conclude that $r$ is more important than they had thought when standard are too high in a typical equilibrium. The unexpectedly rejected papers are relatively low $q$ papers and hence a steeper line allows referees to account for many of the unexpected rejections while maintaining a good fit to the $r(q)$ data for high $q$ papers. I have not discussed this case in more detail because in a couple of ways the argument here seems less plausible. First, it involves economists mistakenly not achieving levels of 
$r$-quality that they could have achieved. Second, the effect derives from economists trying to fit datapoints with $r(q)$ negative.

\section{The overconfidence bias and gradual evolution}

In this section I show that adding a slight overconfidence bias produces a model in which social norms can slowly and steadily evolve over a long period to place ever more emphasis on $r$-quality.

\section{1 $\epsilon$-perturbations of dynamics with a continuum of steady-states}

Before discussing the model, it is instructive to discuss its stucture in more generality. The dynamic model is of the form

$$
\left(\begin{array}{c}
\alpha_{t+1}-\alpha_{t} \\
z_{t+1}-z_{t}
\end{array}\right)=k\left(\begin{array}{c}
\hat{\alpha}_{t}-\alpha_{t} \\
\hat{z}_{t}-z_{t}
\end{array}\right)
$$

where $(\hat{\alpha}, \hat{z})=\arg \min _{\alpha, z} L\left(\alpha, z ; \alpha_{t}, z_{t}\right){ }^{17}$ A social norm $\left(\alpha_{t}, z_{t}\right)$ is a steady-state only if it is a solution to

$$
\begin{aligned}
& \frac{\partial L}{\partial \alpha}\left(\alpha_{t}, z_{t} ; \alpha_{t}, z_{t}\right)=0 \\
& \frac{\partial L}{\partial z}\left(\alpha_{t}, z_{t} ; \alpha_{t}, z_{t}\right)=0
\end{aligned}
$$

This is a system of two equations in two unknowns. Ordinarily, one would expect such a system to have one solution (or zero or a few). The fact that the dynamic model of the previous section has a continuum of equilibria indicates that it is somewhat special.

What happens if we take a dynamic model with a continuum of equilibria and perturb it slightly? The answer of course depends on how the system is pertubed. To take a simple example, consider a dynamic of the form above with the loss function

$$
L\left(\alpha, z ; \alpha_{t}, z_{t}\right)=\left(\alpha-\alpha_{t}\right)^{2}+\left(z-z_{t}\right)^{2}+(z-\alpha)^{2} .
$$

This model has every point on the line $z_{t}=\alpha_{t}$ as a steady state. One thing that can happen with an $\epsilon$-perturbation is an $\epsilon$-order shift in the set of steady states. For example,

\footnotetext{
${ }^{17}$ While I earlier defined $L$ as depending on the measures $\mu_{1}$ and $\mu_{2}$ describing the two types of data, $\mu_{1}$ and $\mu_{2}$ are themselves functions of $\alpha_{t}$ and $z_{t}$. I use $\alpha_{t}$ and $z_{t}$ as arguments of $L$ in this section to clarify the nature of the dynamic.
} 
if we perturb the loss function above to

$$
L\left(\alpha, z ; \alpha_{t}, z_{t}, \epsilon\right)=\left(\alpha-\alpha_{t}\right)^{2}+\left(z-z_{t}\right)^{2}+(z-(\alpha+\epsilon))^{2},
$$

the system has a continuum of steady states given by $z_{t}=\alpha_{t}+\epsilon$.

With a generic perturbation, however, the continuum of steady states will disappear. For example, if we instead perturb the system to

$$
L\left(\alpha, z ; \alpha_{t}, z_{t}, \epsilon\right)=\left(\alpha-\alpha_{t}\right)^{2}+\left(z-z_{t}\right)^{2}+(z-\alpha)^{2}+\epsilon z^{2},
$$

the only remaining steady-state is $\alpha_{t}=z_{t}=0$. What happens to the former equilibria? In this example, the $\epsilon$ perturbed dynamics are

$$
\begin{aligned}
\hat{\alpha}-\alpha_{t} & =\frac{1+\epsilon}{3+2 \epsilon}\left(z_{t}-\alpha_{t}\right)-\frac{\epsilon}{3+2 \epsilon}\left(\alpha_{t}+z_{t}\right) \\
\hat{z}-z_{t} & =\frac{1+\epsilon}{3+2 \epsilon}\left(\alpha_{t}-z_{t}\right)-\frac{\epsilon}{3+2 \epsilon}\left(\alpha_{t}+z_{t}\right)
\end{aligned}
$$

The first terms on the right hand sides of these equations tell us that from any initial condition the dynamics lead quickly to a neighborhood of the nearly stable locus $z_{t}=\alpha_{t}$. The system then evolves at an $\epsilon$ rate in a neighborhood of this locus toward the steady state. If the initial condition is very from the steady state a long gradual evolution would be observed.

Obviously, the most natural way to account for a long gradual trend in an economic variable will usually be to view the trend as reflecting a continuous shift in the equilibrium of a model due to a trending exogenous variable. The most general idea the dynamic model is intended to convey is that the disequilibrium dynamics in perturbations of models with a continuum of equilibria may provide an alternate method for explaining some such trends.

\subsection{Social norms for academic publishing}

My main observation about the model with no overconfidence bias was that if referees try to hold authors to an unreasonably high standard, then social norms will evolve to place more weight on $r$-quality. This disequilibrium shift is not an appealing candidate for explaining observed shifts in academic standards for two reasons. First, such an explanation would be incomplete in that we have no reason to expect initial beliefs to be too demanding as 
opposed to too undemanding. Second, and more importantly, the model does not fit a long gradual trend. Instead, social norms in such a model move very quickly to a neighborhood of the equilibrium set and then converge slowly without much further movement along the equilibrium set.

The dynamics with an $\epsilon$ overconfidence bias are qualitatively different. When beliefs are far from the equilibrium set, the $\epsilon$-perturbation has little impact — we again see rapid convergence to a neighborhood of the set of equilibria of the unperturbed model. The overconfidence then becomes important. In the typical case it destabilizes the former equilibria. If economists' initial beliefs correspond to a consistent social norm, then their misperceptions of the referee reports they receive will lead them to conclude that overall quality standards must be higher than they had thought (i.e. $\hat{z}-z_{t}>0$.) For this reason, referees will perpetually try to hold authors to a standard that is slightly higher than is feasible. The $\epsilon$-overconfidence does not affect the argument that economists will conclude that $r$-quality is more important than they had thought whenever standards are higher than is feasible. Hence, when the equilibrium has the typical form we will observe a gradual evolution of social norms through the near equilibrium set toward lower $\alpha$ values.

Must we look forward to a world with no q-quality whatsoever? In my model at least, the answer is no. In the neighborhood of a consistent social norm the effect of a small overconfidence bias is to make economists think that $z$ must be slightly higher than they had thought. In the low $\alpha$ and somewhat low $\alpha$ cases, when the standard is slightly too high economists infer that $z$ must be slightly lower than they had thought. For any such $\alpha$, the two effects turn out to exactly offset for some $z$ slightly greater than $z^{*}(\alpha)$. The $\epsilon$-perturbed model has a continuum of steady-states lying just above the low $\alpha$ and somewhat low $\alpha$ portions of the set of steady states of the unperturbed model. The model thus predicts that the evolution of social norms will come to a halt as soon as $r$ is sufficiently important so that no paper's idea is good enough to let its author be sure that he or she will eventually be able to get an acceptance.

Figure 8 illustrates the dynamics of the system with a small overconfidence bias. The figure was generated by solving the model numerically for various initial beliefs under the assumption that $q \sim U\left[0, t_{q}\right], \tau=0.3, \sigma=0.2, h(t)=\sqrt{t}-t / 2$ and $\epsilon=0.01$. The thick 
solid curve on the left side of the figure is the locus of steady states $\left(\alpha, z^{\epsilon}(\alpha)\right)$. On the right side of the figure I've graphed eight curves illustrating the evolution of social norms from eight initial conditions. While the curves appear to join together well before they reach the left endpoint, they actually remain separate over the whole range and in an extremely magnified figure what now appears to be one central curve would be revealed to be a set of nearly parallel curves. From initial conditions with $z$ large the system initially evolves down and to the left toward the set of nearly stable norms and then moves along this set until the somewhat low $\alpha$ region is reached. From initial conditions with $z$ small there is an initial evolution up toward the nearly stable set, and then again a movement to the left along the nearly stable set.

The arrows on the curves give a feeling for the speed of movement. The arrows mark the beliefs that prevail after one period of evolution and then after every ten additional periods. ${ }^{18}$ The fact that the arrows are initially far apart on each curve reflects that the initial movement toward the nearly stable set is rapid - from most starting points the curves get sufficiently close to the nearly stable set to be indistinguishable to the naked eye within ten or twenty periods. The fact that arrows then become closely and regularly spaced as norms evolve along the nearly stable set reflects that this evolution is slow and steady. Here, the evolution from a near equilibrium with $\alpha=0.8$ to a near equilibrium with $\alpha=0.4$ takes about two hundred periods.

Proposition 7 provides a formal description of some properties of the system. Part (a) notes that the model has a continuum of equilibria covering roughly the low $\alpha$ and somewhat low $\alpha$ ranges. Part (b) notes that when $\alpha$ is such that the equilibrium has the typical form there is no steady state: at $\left(\alpha, z^{*}(\alpha)\right)$ the data is better fit by increasing $z$; when $z$ is slightly higher than $z^{*}(\alpha)$ the fit is improved by reducing $\alpha$.

Proposition 7 Consider the dynamic model described above with $F\left(q \mid t_{q}\right) \sim U\left[0, t_{q}\right]$. Let $t_{q}(\alpha, z)$ be economists' optimal time allocation when they believe that the social norm is $(\alpha, z)$.

(a) Suppose that for a given $\alpha \in(0,1)$ the unique equilibrium for the social norm $\left(\alpha, z^{*}(\alpha)\right)$ with $\epsilon=0$ has the low $\alpha$ or somewhat low $\alpha$ form. Suppose that $\frac{\partial^{2} G}{\partial t^{2}}$ is strictly positive

\footnotetext{
${ }^{18}$ In constructing the figure I took $k=0.5$
} 


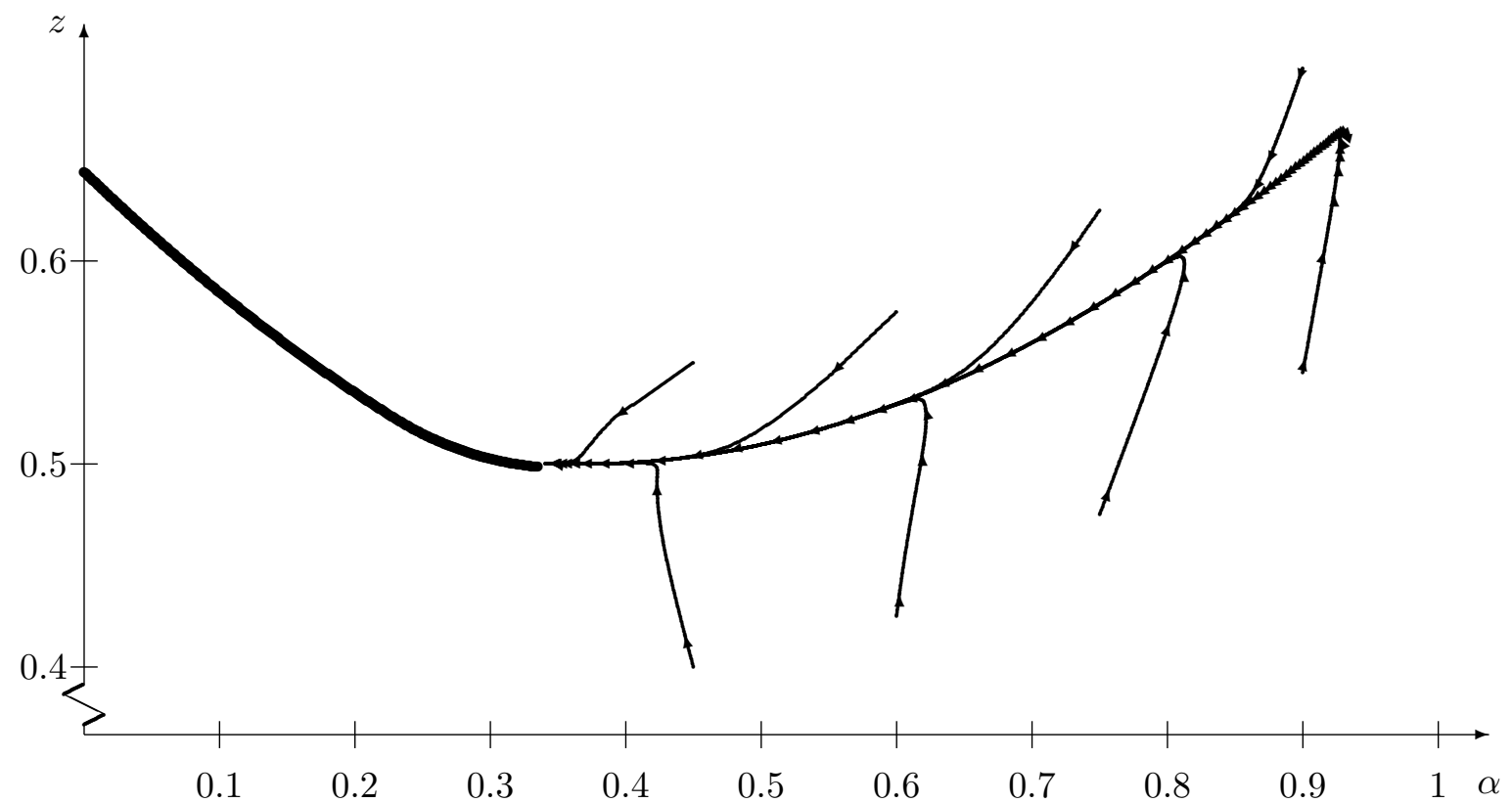

Figure 8: The disequilibrium evolution of social norms when $\epsilon>0$.

at $t=t_{q}\left(\alpha, z^{*}(\alpha)\right)$. Then for $\epsilon$ sufficiently small there exists a value of $z, z^{\epsilon}(\alpha)$ such that $\left(\alpha, z^{\epsilon}(\alpha)\right)$ is a steady state of the model with $\epsilon$-overconfidence

Further, there exists a positive constant a such that, $z^{\epsilon}(\alpha)-z^{*}(\alpha) \approx a \epsilon$ when $\epsilon$ is small. In the low $\alpha$ case $z^{\epsilon}(\alpha)$ is exactly equal to $z^{*}(\alpha)+2(1-\alpha) \sigma \epsilon$ when $\epsilon$ is sufficiently small.

(b) Suppose that for a given $\alpha \in(0,1)$ the unique equilibrium for the social norm $\left(\alpha, z^{*}(\alpha)\right)$ with $\epsilon=0$ has the typical form. When $\epsilon>0$ we have $\frac{\partial L}{\partial z}\left(\alpha, z^{*}(\alpha) ; \alpha, z^{*}(\alpha)\right)<0$. For $\epsilon$ sufficiently small there exists a $z>z^{*}(\alpha)$ for which the distribution of paper qualities and expectedly and unexpectedly accepted papers has the typical form pictured in Figure 6 and the loss function has $\frac{\partial L}{\partial z}(\alpha, z ; \alpha, z)=0$. For any such $z, \frac{\partial L}{\partial \alpha}(\alpha, z ; \alpha, z)<0$. Hence, the system does not have a steady state where the distribution of paper qualities has the typical form.

Let $z^{\epsilon}(\alpha)$ be the smallest value of $z$ such that $\frac{\partial L}{\partial z}(\alpha, z ; \alpha, z)=0$. Then there exist positive constants a and $b$ such that $z^{\epsilon}(\alpha)-z^{*}(\alpha) \approx a \epsilon$ and $\frac{\partial L}{\partial \alpha}\left(\alpha, z^{\epsilon}(\alpha) ; \alpha, z^{\epsilon}(\alpha)\right) \approx-b \epsilon$ for small $\epsilon$.

For those who do not believe that academics suffer from overconfidence bias (and those 
who are wondering if observed changes in standards should be taken as providing evidence of such a bias), I would like to note that a gradual evolution toward lower $\alpha$ norms can be generated by many small modifications to the basic dynamic model. Essentially, any perturbation that makes referees perpetually try to hold authors to a slightly infeasibly high standard will do the same thing. One example would be an assumption that referees try to make up more extensive lists of revisions than is standard to impress editors with their thoroughness. Another would be an assumption that referees are competitive (or spiteful) and try to hold back others in their field by imposing standards that are slightly higher than the norm (perhaps because making requests that are even more demanding would expose their spite).

In a standard one-dimensional model where the optimal level of revisions is determined by a cost of revisions or in a one-dimensional learning model where referees are learn $z$ by observing referees' reports and editors' decisions, an $\epsilon$-perturbation like these just produces a model in which the original equilibrium (or steady-state) is shifted by $\epsilon$. In the twodimensional learning model, however, $\epsilon$ changes can greatly change the equilibrium set and create steady long-term disequilibrium evolution.

\section{Conclusion}

I have proposed that it is helpful to think about observed changes in the economics publishing process with a two-dimensional $q-r$ model of quality. The idea behind the dynamic model is that social norms for weighing different aspects of quality may shift as economists struggle to reconcile the high standards being applied to them with the mediocrity they see in journals. The model predicts a long gradual shift in standards. Academics react

by spending less and less time developing new insights and more and more time polishing papers.

Among the many possible extensions to the model, I view the incorporation of randomness in the assessment of quality and in economists' data samples as the most intriguing. Referees who receive signals suggesting that standards are higher than they are may conclude that $r$-quality is more important than they thought, while referees who receive signals suggesting that standards are lower than they are will not draw the opposite conclusion. 
Even without an overconfidence bias we may see increasing emphasis placed on $r$-quality. Sobel's (1998) work suggests that randomness in assessments due to heterogeneity in tastes may have more subtle effects.

What are the welfare effects of changes in publication standards? There are no answers within my model, because individual preferences over $q$ and $r$ do not appear. If preferences are time invariant, then welfare changes as standards evolve. Whether changes are welfare increasing or welfare decreasing depends on how the prevailing social norm differs from the optimum given authors' and readers' preferences.

The changes of the last few decades have had a substantial impact on economists. Many young economists report spending as much time revising old papers as working on new ones. Guiding larger and more frequent revisions is an additional burden on referees and editors. If the changes that have occurred were accidental and many other social norms are really possible, then a discussion in the profession of what standards we would like to have would be valuable.

While I have emphasized the model of learning with overconfidence, other explanations for the observed changes are possible. Indeed, one of the main motivations for the static model is to clarify how the growth of the economics profession, changes in the set of ideas waiting to be discovered and other factors might alter the publication process. Ultimately, which of the potential explanations are most important is an empirical question. I attempt to begin this sorting out in Ellison (2000).

How else might one examine the evolutionary model empirically? With ideal data one could directly examine referees' demands and see how they are affected by referees' experiences as authors. A general premise of the model that may be more easily examined is that learning occurs within communities of referees. A good empirical test would be to observe whether a random shock to a particular field at a particular journal, e.g. the appointment of a new editor who likes revisions, affects how papers in the field are treated at other journals. Another feature that distinguishes the evolutionary model from equilibrium models is that transitory shocks have permanent effects. For example, even after a revisionloving editor is replaced, the fact that referees learned a high revision norm will ensure that publication remains slow for years to come. 
In addition to trying to change how people think about academic publishing, I have tried to make the general point that a long run trend can be a disequilibrium phenomenon. Comparative statics of equilibria will remain the standard for explaining trends, but I hope that models like that I've developed here will find other applications. 


\section{Appendix A}

Proof of Proposition 6

(a) In the low $\alpha$ case for $\left(z, t_{q}\right)$ in a neighborhood of $\left(z^{*}\left(\alpha_{t}\right), t_{q}\left(\alpha_{t}, z^{*}\left(\alpha_{t}\right)\right)\right)$ we have

$$
G\left(z, t_{q}\right)=\frac{1}{\sigma}\left(\frac{z-\alpha_{t} t_{q} / 2}{1-\alpha_{t}}-h\left(1-t_{q}\right)\right) .
$$

This function has $\frac{\partial G}{\partial t_{q}}=-\frac{\alpha_{t}}{2 \sigma\left(1-\alpha_{t}\right)}+h^{\prime}\left(1-t_{q}\right)$, which is independent of $z$. It also has $\frac{\partial^{2} G}{\partial t_{q}^{2}}=-h^{\prime \prime}\left(1-t_{q}\right)>0$, and hence in a neighborhood of $z^{*}\left(\alpha_{t}\right), t_{q}\left(\alpha_{t}, z_{t}\right)$ is independent of $z_{t}$. The continuity of the thresholds implies that the ordering $\underline{q}<0<m\left(t_{q}\right)<\bar{q}$ holds for all $z_{t}$ in a neighborhood of $z^{*}\left(\alpha_{t}\right)$.

Write $r_{a}\left(q ; \alpha_{t}, z_{t}\right)$ for the level of $r$-quality that is actually necessary for an editor to accept a paper of $q$-quality $q$ when the population believes the standard is $\left(\alpha_{t}, z_{t}\right)$. Because $t_{q}\left(\alpha_{t}, z_{t}\right)$ is locally independent of $z_{t}$ and all authors revise to the greatest extent possible, $r_{a}\left(q ; \alpha_{t}, z_{t}\right)=r\left(q ; \alpha_{t}, z^{*}\left(\alpha_{t}\right)\right)$ for $z_{t}$ near $z^{*}\left(\alpha_{t}\right)$. The situation is thus like that pictured in Figure 7. When $z_{t}<z^{*}\left(\alpha_{t}\right)$ the situation is similar, but involving a parallelogram of unexpectedly rejected papers lying just above the $(q, r(q))$ line.

To describe economists' inferences, I'll make a change of variables and analyze the minimization of the loss function over $m$ and $w$ where $m=\frac{-\alpha}{1-\alpha}$ is the slope of the line and $w=\frac{z-\alpha M_{q}}{1-\alpha}$ is the level of $r$ quality required of a paper with the mean $q$-quality, $M_{q} \equiv\left(t_{q}\left(\alpha_{t}, z_{t}\right)+\max \left(0, \underline{q}\left(\alpha_{t}, z_{t}\right)\right)\right) / 2$. I'll carry out the calculations for $z_{t}>z^{*}\left(\alpha_{t}\right)$. The calculations in the opposite case are identical but with some signs reversed.

With the change of variables $L_{1}$ takes on a very simple form. ${ }^{19}$

$$
\begin{aligned}
L_{1}(m, w) & =\int_{0}^{t_{q}\left(\alpha_{t}, z_{t}\right)}\left(m\left(q-M_{q}\right)+w-r\left(q ; \alpha_{t}, z_{t}\right)\right)^{2} \frac{1}{t_{q}\left(\alpha_{t}, z_{t}\right)} d q \\
& =\frac{1}{t_{q}\left(\alpha_{t}, z_{t}\right)} \int_{0}^{t_{q}\left(\alpha_{t}, z_{t}\right)}\left(m\left(q-M_{q}\right)+w-\left(m_{t}\left(q-M_{q}\right)+w_{t}\right)\right)^{2} d q \\
& =\frac{1}{t_{q}\left(\alpha_{t}, z_{t}\right)}\left[\left(m-m_{t}\right)^{2} \frac{\left(q-M_{q}\right)^{3}}{3}+\left(m-m_{t}\right)\left(w-w_{t}\right)\left(q-M_{q}\right)^{2}+\left(w-w_{t}\right)^{2} q\right]_{0}^{t_{q}\left(\alpha_{t}, z_{t}\right)} \\
& =\frac{1}{12} t_{q}\left(\alpha_{t}, z_{t}\right)^{2}\left(m-m_{t}\right)^{2}+\left(w-w_{t}\right)^{2}
\end{aligned}
$$

By making a few comparisons it is easy to see that the minimum of $L_{1}+L_{2}$ can not be achieved for $w>w_{t}$, or for $w<r_{a}\left(M_{q}, \alpha_{t}, z_{t}\right)$, or with a slope $m$ for which $w+m\left(-M_{q}\right)$ is outside the interval $\left[r_{a}\left(0 ; \alpha_{t}, z_{t}\right), r\left(0 ; \alpha_{t}, z_{t}\right)\right]$. (For example, to show the second note that for any such $w, L(m, w)>L\left(m_{t}, r_{a}\left(M_{q}, \alpha_{t}, z_{t}\right)\right)$ because $L_{1}$ is smaller at $\left(m_{t}, r_{a}\left(M_{q}, \alpha_{t}, z_{t}\right)\right)$ and $L_{2}$ has its global minimum there.) In the range containing any potential minumum, $L_{2}$ turns out to also be a simple quadratic.

$$
L_{2}(m, w)=\int_{0}^{t_{q}\left(\alpha_{t}, z_{t}\right)} \int_{0}^{w-r_{a}\left(M_{q} ; \alpha_{t}, z_{t}\right)+\left(m-m_{t}\right)\left(q-M_{q}\right)} r \frac{1}{\sigma} d r \frac{1}{t_{q}\left(\alpha_{t}, z_{t}\right)} d q
$$

\footnotetext{
${ }^{19}$ To make the formulas more readable I'll often omit the $\mu_{1}$ and $\mu_{2}$ arguments of $L_{1}$ and $L_{2} \cdot \mu_{1}$ and $\mu_{2}$ are determined by $\alpha_{t}$ and $z_{t}$, so I'll also sometimes substitute $\alpha_{t}$ and $z_{t}$ (or $m_{t}$ and $w_{t}$ ) as arguments. I will also omit arguments of $M_{q}, t_{q}, \bar{q}$, and $\underline{q}$ to improve readability.
} 


$$
\begin{aligned}
& =\frac{1}{2 \sigma t_{q}\left(\alpha_{t}, z_{t}\right)} \int_{0}^{t_{q}\left(\alpha_{t}, z_{t}\right)}\left(w-r_{a}\left(M_{q} ; \alpha_{t}, z_{t}\right)+\left(m-m_{t}\right)\left(q-M_{q}\right)\right)^{2} d q \\
& =\frac{1}{24 \sigma} t_{q}\left(\alpha_{t}, z_{t}\right)^{2}\left(m-m_{t}\right)^{2}+\frac{1}{2 \sigma}\left(w-r_{a}\left(M_{q} ; \alpha_{t}, z_{t}\right)\right)^{2} .
\end{aligned}
$$

Hence,

$$
L\left(m, w ; m_{t}, w_{t}\right)=\frac{1}{6} t_{q}\left(\alpha_{t}, z_{t}\right)^{2}\left(m-m_{t}\right)^{2}+\left(w-w_{t}\right)^{2}+\frac{1}{2 \sigma}\left(w-r_{a}\left(M_{q} ; \alpha_{t}, z_{t}\right)\right)^{2} .
$$

The minimum clearly involves $\hat{m}=m_{t}$. This gives the first part of the conclusion: $\hat{\alpha}=\alpha_{t}$.

The minimizing value for $w$ is $\hat{w}=\frac{2 w_{t}+(1 / \sigma) r_{a}\left(M_{q} ; \alpha_{t}, z_{t}\right)}{2+1 / \sigma}$. Recall that $r_{a}$ is independent of $z_{t}$ in a neighborhood of $z^{*}\left(\alpha_{t}\right)$. We thus have

$$
\begin{aligned}
r_{a}\left(M_{q} ; \alpha_{t}, z_{t}\right) & =\frac{z^{*}\left(\alpha_{t}\right)-\alpha_{t} M_{q}}{1-\alpha_{t}} \\
& =\frac{z^{*}\left(\alpha_{t}\right)-z_{t}}{1-\alpha_{t}}+\frac{z_{t}-\alpha_{t} M_{q}}{1-\alpha_{t}}=\frac{z^{*}\left(\alpha_{t}\right)-z_{t}}{1-\alpha_{t}}+w_{t} .
\end{aligned}
$$

Substituting this into the expression for $\hat{w}$ gives

$$
\hat{w}-w_{t}=\frac{1}{(2 \sigma+1)\left(1-\alpha_{t}\right)}\left(z^{*}\left(\alpha_{t}\right)-z_{t}\right) .
$$

Using the identities $\alpha_{t}=\hat{\alpha}$ and $z_{t}=\alpha_{t} M_{q}+\left(1-\alpha_{t}\right) w_{t}$ allows us to conclude as desired that

$$
\begin{aligned}
z_{t+1}-z_{t} & =k\left(\hat{\alpha} M_{q}+(1-\hat{\alpha}) \hat{w}-z_{t}\right)=k\left(\alpha_{t} M_{q}+\left(1-\alpha_{t}\right) \hat{w}-\alpha_{t} M_{q}+\left(1-\alpha_{t}\right) w_{t}\right) \\
& =k\left(1-\alpha_{t}\right)\left(\hat{w}-w_{t}\right)=k \frac{1}{2 \sigma+1}\left(z^{*}\left(\alpha_{t}\right)-z_{t}\right) .
\end{aligned}
$$

(b) The proof for the somewhat low $\alpha$ case is very similar. One difference is that the form of $G$ is different. This makes it possible that the second derivative of $G$ will not be strictly positive at $t_{q}\left(\alpha_{t}, z^{*}\left(\alpha_{t}\right)\right)$, in which case $t_{q}\left(\alpha_{t}, z_{t}\right)$ might not be differentiable at $z_{t}=z^{*}\left(\alpha_{t}\right)$. This would cause many complications. To avoid them, I've just assumed in the proposition that $\frac{\partial^{2} G}{\partial t_{q}^{2}}\left(\alpha_{t}, t_{q}\left(\alpha_{t}, z^{*}\left(\alpha_{t}\right)\right)\right)>0$. This ensures that $t_{q}\left(\alpha_{t}, z\right)$ is differentiable in $z$ at $z^{*}\left(\alpha_{t}\right)$.

The analysis of the $z_{t}>z^{*}\left(\alpha_{t}\right)$ case then proceeds exactly as above to show that

$$
\begin{aligned}
\hat{\alpha}-\alpha_{t} & =0 \\
\hat{w}-w_{t} & =\frac{1}{2 \sigma+1}\left(r_{a}\left(M_{q} ; \alpha_{t}, z_{t}\right)-w_{t}\right) .
\end{aligned}
$$

Write $\tau_{a}\left(z_{t}\right)=1-G\left(z_{t} ; t_{q}\left(\alpha_{t}, z_{t}\right)\right)$ for the fraction of papers achieving the $z_{t}$ standard given the inital beliefs. By the envelope theorem we know that in a neighborhood of $z^{*}\left(\alpha_{t}\right)$,

$$
\begin{aligned}
\tau_{a}\left(z_{t}\right) & \approx \tau-\frac{\partial G}{\partial z}\left(z^{*}\left(\alpha_{t}\right) ; t_{q}\left(\alpha_{t}, z^{*}\left(\alpha_{t}\right)\right)\left(z_{t}-z^{*}\left(\alpha_{t}\right)\right)\right. \\
& =\tau-\frac{t_{q}\left(\alpha_{t}, z^{*}\left(\alpha_{t}\right)\right)-\underline{q}\left(\alpha_{t}, z^{*}\left(\alpha_{t}\right)\right)}{\left(1-\alpha_{t}\right) \sigma t_{q}\left(\alpha_{t}, z^{*}\left(\alpha_{t}\right)\right)}\left(z_{t}-z^{*}\left(\alpha_{t}\right)\right)
\end{aligned}
$$


The mass of extra papers the editor accepts when he lowers the standard from $z^{*}\left(\alpha_{t}\right)$ to $z^{*}\left(\alpha_{t}\right)-d z$ is $\frac{t_{q}-\underline{q}}{t_{q}\left(1-\alpha_{t}\right) \sigma} d z$. Hence, in a neighborhood of $z^{*}\left(\alpha_{t}\right)$ we have

$$
\begin{aligned}
r_{a}\left(M_{q} ; \alpha_{t}, z_{t}\right)-w_{t} & =\frac{\left(1-\alpha_{t}\right) \sigma t_{q}}{\left(1-\alpha_{t}\right)\left(t_{q}-\underline{q}\right)}\left(\tau_{a}\left(z_{t}\right)-\tau\right) \\
& \approx-\frac{1}{\left(1-\alpha_{t}\right)}\left(z_{t}-z^{*}\left(\alpha_{t}\right)\right)
\end{aligned}
$$

This gives

$$
z_{t+1}-z_{t} \approx a\left(z^{*}\left(\alpha_{t}\right)-z_{t}\right)
$$

for $a=\frac{k}{2 \sigma+1}$ as desired.

The analysis of the $z_{t}<z^{*}\left(\alpha_{t}\right)$ case adds another slight complication - the formula for $L_{2}$ is slightly different because there are no papers in the triangle bounded by $(\underline{q}, h(1-$ $\left.\left.t_{q}\right)+\sigma\right),\left(\underline{q}, r_{a}\left(\underline{q}, \alpha_{t}, z_{t}\right)\right)$, and $\left(\underline{q}+\left(1-\alpha_{t}\right)\left(r_{a}\left(\underline{q}, \alpha_{t}, z_{t}\right)-r\left(\underline{q}, \alpha_{t}, z_{t}\right)\right) / \alpha_{t}, h\left(1-t_{q}\right)+\sigma\right)$ that can be unexpectedly rejected. The loss function thus takes the form

$$
\begin{aligned}
L\left(m, w ; m_{t}, w_{t}\right)= & \frac{2 \sigma+1}{24 \sigma}\left(t_{q}-\underline{q}\right)^{2}\left(m-m_{t}\right)^{2}+\left(w-w_{t}\right)^{2}+\frac{1}{2 \sigma}\left(w-r_{a}\left(M_{q} ; \alpha_{t}, z_{t}\right)\right)^{2} \\
& -\frac{1}{\sigma\left(t_{q}-\underline{q}\right)} \int_{T} I(r>r(q ; m, w))(r-r(q ; m, w)) d q d r,
\end{aligned}
$$

where $T$ is the triangle bounded by the three points above and $I$ is the indicator function. To show that $\alpha_{t+1}-\alpha_{t} \approx 0$ it suffices to show that for any $c>0$ there exists a $\delta$ such that $\left|\hat{m}-m_{t}\right|<c\left(z^{*}\left(\alpha_{t}\right)-z_{t}\right)$ whenever $z_{t} \in\left(z^{*}\left(\alpha_{t}\right)-\delta, z^{*}\left(\alpha_{t}\right)\right)$. To see this note that for all $z_{t}$ in some interval below $z^{*}\left(\alpha_{t}\right)$ we have for any $m<m_{t}-c\left(z^{*}\left(\alpha_{t}\right)-z_{t}\right)$

$$
\begin{aligned}
\frac{\partial L}{\partial m}(m, w)= & \frac{2 \sigma+1}{12 \sigma}\left(t_{q}-\underline{q}\right)^{2}\left(m-m_{t}\right)-\frac{1}{\sigma\left(t_{q}-\underline{q}\right)} \frac{\partial}{\partial m} \int_{T} I(r>r(q ; m, w))(r-r(q ; m, w)) d q d r \\
< & -\frac{2 \sigma+1}{12 \sigma}\left(t_{q}-\underline{q}\right)^{2} c\left(z^{*}\left(\alpha_{t}\right)-z_{t}\right) \\
& \quad+\frac{A(T)}{\sigma\left(t_{q}-\underline{q}\right)} \sup _{(q, r) \in T}\left|\frac{\partial}{\partial m} I(r>r(q ; m, w))(r-r(q ; m, w))\right|,
\end{aligned}
$$

where $A(T)$ is the area of the triangle $T$. The area of the triangle is $\frac{1-\alpha_{t}}{2 \alpha_{t}}\left(r_{a}\left(q ; \alpha_{t}, z_{t}\right)-\right.$ $\left.r\left(q ; \alpha_{t}, z_{t}\right)\right)^{2}$. The effect of a $d m$ change in $m$ on $r(q ; m, w)$ is $q-M_{q}$, which is largest when $q$ is farthest from $M_{q}$. This gives

$$
\frac{\partial L}{\partial m}(m, w) \leq-\frac{2 \sigma+1}{12 \sigma}\left(t_{q}-\underline{q}\right)^{2} c\left(z^{*}\left(\alpha_{t}\right)-z_{t}\right)+\frac{1-\alpha_{t}}{4 \alpha_{t} \sigma}\left(r_{a}\left(q ; \alpha_{t}, z_{t}\right)-r\left(q ; \alpha_{t}, z_{t}\right)\right)^{2} .
$$

The $\left(r_{a}\left(q ; \alpha_{t}, z_{t}\right)-r\left(q ; \alpha_{t}, z_{t}\right)\right)^{2}$ term is a second order effect in $z^{*}\left(\alpha_{t}\right)-z_{t}$. Hence, $\frac{\partial L}{\partial m}(m, w)<$ 0 for all $m<m_{t}-c\left(z^{*}\left(\alpha_{t}\right)-z_{t}\right)$ when $z_{t}$ sufficiently close to $z^{*}\left(\alpha_{t}\right)$. Combining this with a similar calculation of the derivative for $m>m_{t}+c\left(z^{*}\left(\alpha_{t}\right)-z_{t}\right)$ allows us to conclude that $\hat{m} \in\left(m_{t}-c\left(z^{*}\left(\alpha_{t}\right)-z_{t}\right), m_{t}+c\left(z^{*}\left(\alpha_{t}\right)-z_{t}\right)\right)$ as desired.

A similar calculation shows that the result on $z_{t+1}-z_{t}$ is also unaffected by the second order change in the loss function. 
(c) When the equilibrium has the typical form $G\left(z ; t_{q}\right)$ is given by

$$
G\left(z ; t_{q}\right)=\frac{z-\left(1-\alpha_{t}\right)\left(h\left(1-t_{q}\right)+\sigma / 2\right)}{\alpha_{t} t_{q}}
$$

An explicit calculation of the first two derivatives of this function shows that $\frac{\partial^{2} G}{\partial t_{q}^{2}}\left(z ; t_{q}\right)>0$ in a neighborhood of $\left(z^{*}\left(\alpha_{t}\right), t_{q}\left(\alpha_{t}, z^{*}\left(\alpha_{t}\right)\right)\right)$. This again implies that $\frac{\partial t_{q}}{\partial z}\left(\alpha_{t}, z^{*}\left(\alpha_{t}\right)\right)$ exists and that the distribution of paper qualities has the typical form for $z_{t}$ in some neighborhood of $z^{*}\left(\alpha_{t}\right)$.

The $(q, r(q))$ line and the set of unexpectedly rejected papers have exactly the same form here as in $z_{t}<z^{*}\left(\alpha_{t}\right)$ case of part (b) of the proposition. The result is thus identical to the result for that case.

(d) As above $t_{q}$ is differentiable in $z$ at $z^{*}\left(\alpha_{t}\right)$. The distribution of paper qualities and outcomes has the typical form pictured in Figure 6 for all $z_{t}$ in some neighborhood $\left(z^{*}\left(\alpha_{t}\right), z^{*}\left(\alpha_{t}\right)+\right.$ $\delta)$.

Write $w_{a}$ for $r_{a}\left(M_{q} ; \alpha_{t}, z_{t}\right)$. I first note that a number of simple comparisons imply that economists must infer that standards are lower and $q$ is less important than they had thought. Specifically, we must have $\hat{m}>m_{t}$ and $\hat{w} \in\left(w_{a}, w_{t}\right)$. To see this, note first that the line given by $(\hat{m}, \hat{w})$ can not be entirely above the $\left(m_{t}, w_{t}\right)$ line over the whole range $\left[q\left(\alpha_{t}, z_{t}\right), t_{q}\left(\alpha_{t}, z_{t}\right)\right]$ of $q$-qualities of resubmitted papers. From any such estimate both $L_{1}$ and $L_{2}$ are decreased by moving the line down until there is an intersection. Next, note that it is also impossible for the minimum to have $\hat{w}>w_{t}$ with the $(\hat{m}, \hat{w})$ line intersecting the $\left(m_{t}, w_{t}\right)$ line at $q \in\left[\underline{q}, t_{q}\right]$. In this case, $L_{1}$ and $L_{2}$ are both reduced by rotating the fitted line about the point were it intersects the line $r=r_{a}\left(q ; m_{t}, w_{t}\right)$ in the direction that reduces $\hat{w}$. The estimates also can not have $\hat{w}<w_{a}$. In this case increasing $\hat{w}$ to $w_{a}$ and setting $\hat{m}=m_{t}$ reduces $L_{1}$ and makes $L_{2}$ equal to its global minimum. This gives $\hat{w} \in\left(r_{a}\left(M_{q} ; \alpha_{t}, z_{t}\right), w_{t}\right)$. It is then easy to see that $\hat{m} \geq m_{t}$. Otherwise, slightly reducing $\hat{m}$ would reduce $L_{1}$ and also reduce $L_{2}$. (The latter is because the gain from to improving the fit in $\left(q, \min \left(\bar{q}, M_{q}\right)\right)$ is greater than the loss from worsening the fit in the (possibly empty) interval $\left.\left[M_{q}, \bar{q}\right].\right)$ Finally, all of the extreme values $\left(\hat{m}=m_{t}, \hat{w}=w_{t}\right.$ and $\left.\hat{w}=w_{a}\right)$ can be ruled out by looking at derivatives of the loss function.

To obtain the proposition's further conclusion that the changes in $m$ and $w$ are both first order in $z_{t}-z^{*}\left(\alpha_{t}\right)$ I examine the first order conditions of the loss function. Even after the discussion above there remain a few possibilities for exactly where the best fit line may intersect the other lines in the figure: it may be above or below $r_{a}\left(q ; \alpha_{t}, z_{t}\right)$ at the left edge and may be above or below $r\left(t_{q} ; \alpha_{t}, z_{t}\right)$ at the right edge. The first order conditions are slightly different in the four cases. I will work out the equations for the simplest case: assuming that the best fit line is strictly between $r_{a}\left(q ; \alpha_{t}, z_{t}\right)$ and $r\left(q ; \alpha_{t}, z_{t}\right)$ throughout the interval $\left[\underline{q}, t_{q}\right]$.

In this case, the loss function is very similar to the loss function in the cases above. The only differences are that the set of unexpectedly accepted papers does not have papers with $q$-qualities above $\bar{q}$ and is also missing a second-order triangle in $(q, r)$-space below $\left(\bar{q}, r\left(\bar{q} ; \alpha_{t}, z_{t}\right)\right)$. As above, the second-order triangle can be ignored. The estimates have the same asymptotics as those obtained by minimizing loss functions given by integrals identical to those in part (a) but with different lower and upper bounds. Specifically we 
can examine the minimizer of $L=L_{1}+L_{2}$ where

$$
L_{1}(m, w)=\frac{1}{12}\left(t_{q}-\underline{q}\right)^{2}\left(m-m_{t}\right)^{2}+\left(w-w_{t}\right)^{2}
$$

and

$$
L_{2}(m, w)=\frac{1}{24 \sigma} \frac{(\bar{q}-\underline{q})^{3}}{t_{q}-\underline{q}}\left(m-m_{t}\right)^{2}+\frac{1}{2 \sigma} \frac{\bar{q}-\underline{q}}{t_{q}-\underline{q}}\left(w-w_{a}-\left(m-m_{t}\right) \frac{t_{q}-\bar{q}}{2}\right)^{2} .
$$

(Note that I've omitted the arguments $\left(\alpha_{t}, z_{t}\right)$ arguments of $t_{q}, \bar{q}$ and $\underline{q}$ to improve readability.) The first order conditions for this minimization are of the form

$$
\begin{aligned}
c_{1}\left(\hat{m}-m_{t}\right)-c_{2}\left(\hat{w}-w_{a}\right) & =0 \\
-c_{2}\left(\hat{m}-m_{t}\right)+c_{3}\left(\hat{w}-w^{\prime}\right) & =0,
\end{aligned}
$$

where $w^{\prime}=\frac{2 \sigma\left(t_{q}-\underline{q}\right) w_{t}+(\bar{q}-q) w_{a}}{2 \sigma\left(t_{q}-\underline{q}\right)+(\bar{q}-\underline{q})}$ is a weighted average of $w_{t}$ and $w_{a}$ and $c_{1}, c_{2}$ and $c_{3}$ are positive constants: $c_{1}=\frac{1}{6}\left(t_{q}-\underline{q}\right)^{2}+\frac{1}{12 \sigma}\left(\frac{\bar{q}-q}{t_{q}-\underline{q}}\right)^{3}+\frac{1}{4}(\bar{q}-\underline{q})\left(t_{q}-\bar{q}\right) ; c_{2}=\frac{(\bar{q}-q)\left(t_{q}-\bar{q}\right)}{2 \sigma\left(t_{q}-\underline{q}\right)}$; and $c_{3}=2+\frac{\bar{q}-\underline{q}}{\sigma\left(t_{q}-\underline{q}\right)}$. Adding $c_{2} / c_{3}$ times the second equation to the first gives

$$
\hat{m}-m_{t}=\frac{c_{2} c_{3}}{c_{1} c_{3}-c_{2}^{2}} \frac{2 \sigma\left(t_{q}-\underline{q}\right)}{2 \sigma\left(t_{q}-\underline{q}\right)+(\bar{q}-\underline{q})}\left(w_{t}-w_{a}\right) .
$$

We know that the true minimum has $\hat{m}>m_{t}$ and that $w_{t}>w_{a}$, so the first order conditions of this case can only give the true minimum if the constant in this expression is positive. The equations also give

$$
\hat{w}-w_{t}=\frac{c_{2}^{2}-c_{1} c_{3} \frac{\bar{q}-\underline{q}}{2 \sigma\left(t_{q}-\underline{q}\right)+(\bar{q}-q l)}}{c_{1} c_{3}-c_{2}^{2}}\left(w_{t}-w_{a}\right)
$$

From the discussion above this can be the true minumum only if the leading constant is negative.

The fact that $w_{t}-w_{a} \approx\left(z_{t}-z^{*}\left(\alpha_{t}\right)\right) /\left(1-\alpha_{t}\right)$ implies that $\hat{m}-m_{t}$ and $\hat{w}-w_{t}$ are first order in $z_{t}-z^{*}\left(\alpha_{t}\right)$. The longer expression for $z_{t+1}-z_{t}$ follows from the calculation

$$
\begin{aligned}
\hat{z}-z_{t} & =\hat{\alpha} M_{q}+(1-\hat{\alpha}) \hat{w}-\left(\alpha_{t} M_{q}+\left(1-\alpha_{t}\right) w_{t}\right) \\
& =\left(1-\alpha_{t}\right)\left(\hat{w}-w_{t}\right)+M_{q}\left(\hat{\alpha}-\alpha_{t}\right)-\hat{w}\left(\hat{\alpha}-\alpha_{t}\right) \\
& =\left(1-\alpha_{t}\right)\left(\hat{w}-w_{t}\right)+\left(M_{q}-M_{r}\right)\left(\hat{\alpha}-\alpha_{t}\right)+\left(M_{r}-\hat{w}\right)\left(\hat{\alpha}-\alpha_{t}\right)
\end{aligned}
$$

The last term in this expression is second order in $z_{t}-z^{*}\left(\alpha_{t}\right)$.

QED.

\section{Proof of Proposition 7}

(a) Recall from the proof of Proposition 6 that $t_{q}\left(\alpha_{t}, z_{t}\right)$ is differentiable in $z$ at $z^{*}\left(\alpha_{t}\right)$ and that the distribution of paper qualities will also have the low $\alpha$ or somewhat low $\alpha$ form for $z_{t}$ in a neighborhood of $z^{*}\left(\alpha_{t}\right)$. The same computation as in the proof of part (b) of 
Proposition 6 shows that for $z_{t}$ in some neighborhood $\left(z^{*}\left(\alpha_{t}\right), z^{*}\left(\alpha_{t}\right)+\delta\right)$ the loss function is of the form $L=L_{1}+L_{2}$ with

$$
\begin{aligned}
& L_{1}\left(m, w ; m_{t}, w_{t}\right)=\frac{1}{12}\left(t_{q}-\underline{q}\right)^{2}\left(m-m_{t}\right)^{2}+\left(w-\left(w_{t}+\epsilon\right)\right)^{2} \\
& L_{2}\left(m, w ; m_{t}, w_{t}\right)=\frac{1}{24 \sigma}\left(t_{q}-\underline{q}\right)^{2}\left(m-m_{t}\right)^{2}+\frac{1}{2 \sigma}\left(w-r_{a}\left(M_{q} ; \alpha_{t}, z_{t}\right)\right)^{2} .
\end{aligned}
$$

Differentiating, $\left(m_{t}, w_{t}\right)$ is a steady state if and only if $w_{t}-r_{a}\left(M_{q} ; \alpha_{t}, z_{t}\right)=2 \sigma \epsilon$. In the previous proof we also saw that $w_{t}-r_{a}\left(M_{q} ; \alpha_{t}, z_{t}\right) \approx \frac{1}{1-\alpha_{t}}\left(z_{t}-z^{*}\left(\alpha_{t}\right)\right)$. Hence, for $\epsilon$ sufficiently small we can find a $z_{t}>z^{*}\left(\alpha_{t}\right)$ that satisfies the equation for a steady state.

In the somewhat low $\alpha$ case the equations above give $z^{\epsilon}(\alpha)-z^{*}(\alpha) \approx 2(1-\alpha) \sigma \epsilon$. In the low $\alpha$ case we saw earlier that the expression $w_{t}-r_{a}\left(M_{q} ; \alpha_{t}, z_{t}\right)=\frac{1}{1-\alpha_{t}}\left(z_{t}-z^{*}\left(\alpha_{t}\right)\right)$ is exact when $z_{t}$ is sufficiently close to $z^{*}\left(\alpha_{t}\right)$, and hence the expression for $z^{\epsilon}(\alpha)-z^{*}(\alpha)$ is an equality as well.

(b) Again for a given $\alpha_{t}$ the distribution of papers has the typical form if $z_{t}$ is sufficiently close to $z^{*}\left(\alpha_{t}\right)$. For $w_{t}$ in a neighborhood of $r\left(M_{q} ; \alpha_{t}, z_{t}\right)$ and $(m, w)$ sufficiently close to $\left(m_{t}, w_{t}\right)$ with $m \leq m_{t}, w \geq w_{t}$ the expression for the loss function is again a slight variant of that in the previous proposition:

$$
\begin{aligned}
& L_{1}\left(m, w ; m_{t}, w_{t}\right)=\frac{1}{12}\left(t_{q}-\underline{q}\right)^{2}\left(m-m_{t}\right)^{2}+\left(w-\left(w_{t}+\epsilon\right)\right)^{2} \\
& L_{2}\left(m, w ; m_{t}, w_{t}\right)=\frac{1}{24 \sigma}\left(\frac{\bar{q}-\underline{q}}{t_{q}-\underline{q}}\right)^{3}\left(m-m_{t}\right)^{2}+\frac{1}{2 \sigma} \frac{\bar{q}-\underline{q}}{t_{q}-\underline{q}}\left(w-w_{a}-\left(m-m_{t}\right) \frac{t_{q}-\underline{q}}{2}\right)^{2},
\end{aligned}
$$

where again I've written $w_{a}$ for $r_{a}\left(M_{q} ; \alpha_{t}, z_{t}\right)$ to save space. A direct computation shows that $\frac{\partial L}{\partial m}\left(m_{t}, w_{t} ; m_{t}, w_{t}\right)=0$ and $\frac{\partial L}{\partial w}\left(m_{t}, w_{t} ; m_{t}, w_{t}\right)<0$ when $\left(m_{t}, w_{t}\right)$ corresponds to a consistent social norm. This is the first result mentioned in part (b).

We will find $\frac{\partial L}{\partial z}=0$ with $L$ parameterized by $(\alpha, z)$ if and only if $\frac{\partial L}{\partial w}=0$ when $L$ is parameterized by $(m, w)$. A simple calculation of derivatives shows that $\frac{\partial L}{\partial w}\left(m_{t}, w_{t} ; m_{t}, w_{t}\right)=0$ if and only if

$$
w_{t}-w_{a}=2 \sigma \frac{t_{q}-\underline{q}}{\bar{q}-\underline{q}} \epsilon=0
$$

As above this has a solution $z_{t}$ with $z_{t}-z^{*}\left(\alpha_{t}\right) \approx 2\left(1-\alpha_{t}\right) \sigma \frac{t_{q}-q}{\bar{q}-\underline{q}} \epsilon$. This establishes the next claim in part (b) and the fact that the smallest solution has $z^{\bar{\epsilon}}\left(\alpha_{t}\right)-z^{*}\left(\alpha_{t}\right) \approx a \epsilon$ for some $a>0$.

If $\left(m_{t}, w_{t}\right)$ were a steady state of the dynamics in which the distribution of paper qualities had the typical form it would have to satisfy the first-order condition above and the additional constraint that $\frac{\partial L}{\partial m}\left(m_{t}, w_{t} ; m_{t} ; w_{t}\right)=0$. We know that $\frac{\partial L_{1}}{\partial m}\left(m_{t}, w_{t} ; m_{t} ; w_{t}\right)=0$ for any $m_{t}$ and $w_{t}$. The $w$ FOC can only be satisfied for $w_{t}>r\left(M_{q} ; \alpha_{t}, z_{t}\right)$. For such values of $w_{t}$ and for $m \leq m_{t}$ sufficiently close to $m_{t}$ we have

$L_{2}\left(m, w_{t} ; m_{t}, w_{t}\right)=\frac{1}{\sigma\left(t_{q}-\underline{q}\right)}\left(\int_{\max \left(0, \bar{q}-M_{q}\right)}^{\left(t_{q}-\underline{q}\right) / 2} \int_{0}^{\left(m-m_{t}\right) q} r d r d q+\int_{M_{q}-\bar{q}}^{\left(t_{q}-\underline{q}\right) / 2} \int_{0}^{w_{t}-w_{a}-\left(m-m_{t}\right) q} r d r d q\right)$.

The first term is the surprise due to the unexpected acceptance of papers with q-qualities above $\bar{q}$, and the second is the suprise due to the unexpected acceptance of papers with 
lower $q$-qualities. Evaluating the integrals and differentiating gives

$$
\frac{\partial L_{2}}{\partial m}\left(m_{t}, w_{t} ; m_{t}, w_{t}\right)=-\frac{\left(t_{q}-\underline{q}\right)^{2}-4\left(\bar{q}-M_{q}\right)^{2}}{8 \sigma\left(t_{q}-\underline{q}\right)}\left(w_{t}-w_{a}\right) .
$$

The derivative with respect to $\alpha$ is simply this expression divided by $\left(1-\alpha_{t}\right)^{2}$. This is negative, which is the second conclusion of part (b). It is also immediate from the expression that $\frac{\partial L}{\partial \alpha}\left(\alpha_{t}, z_{t} ; \alpha_{t}, z_{t}\right) \approx b \epsilon$.

Looking at the numerator in the equation for $\frac{\partial L}{\partial m}$ we can also see that the gain from reducing $\alpha$ vanishes as we approach the somewhat low $\alpha$ region and $\bar{q}$ approaches $t_{q}$ (or as $\alpha$ approaches one and $\bar{q}$ approaches $\underline{q}$.)

QED. 


\section{References}

De Long, J. Bradford, Andrei Shleifer, Lawrence H. Summers and Robert J. Waldmann (1990): "Noise Trader Risk in Financeial Markets," Journal of Political Economy 98, 703738.

Ellison, Glenn (2000): "The Slowdown of the Economics Publishing Process," mimeo.

Ellison, Glenn and Drew Fudenberg (2000): "Learning Purified Mixed Equilibria," Journal of Economic Theory 90, 84-115.

Karni, Edi and David Schmeidler (1990): "Fixed Preferences and Changing Tastes," American Economic Review 80 (2), 262-267.

Lichtenstein, Sarah, Baruch Fischhoff and Lawrence Phillips (1982): "Calibration of Probabilities: The State of the Art to 1980." In Judgement under Uncertainty: Heuristics and Biases, edited by Daniel Kahneman, Paul Slovic and Amos Tversky. Cambridge: Cambridge University Press.

Odean, Terrance (1998): "Volume, Volatility, Price, and Profit When All Traders Are Above Average." Journal of Finance 53 (6), 1887-1934.

Pesendorfer, Wolfgang (1995): "Design Innovation and Fashion Cycles," American Economic Review 85 (4), 771 - 792.

Rabin, Matthew (1998): "Psychology and Economics," Journal of Economic Literature 36, $11-46$.

Rubinstein, Ariel (1982): "Perfect Equilibrium in a Bargaining Model," Econometrica 50, 97-109.

Sen, Amartya (1970): "The Impossibility of a Paretian Liberal," Journal of Political Economy $78,152-157$.

Sobel, Joel (1998): "On the Dynamics of Standards," University of California at San Diego Discussion Paper 97-15R.

Spence, A. Michael (1973): "Job Market Signaling," Quarterly Journal of Economics 87, 355-374.

Svenson, Ola (1981): "Are We All Less Risky and More Skillful than Our Fellow Drivers," Acta Psychologica 47, 143-148. 
Table 1: Mean references per nonreview article and average page lengths

\begin{tabular}{|c|c|c|c|c|c|}
\hline \multirow[b]{2}{*}{ Field } & \multirow[b]{2}{*}{ Journal } & \multicolumn{2}{|c|}{ Pages } & \multicolumn{2}{|c|}{ References } \\
\hline & & 1975 & 1999 & $1977 / 8$ & 1998 \\
\hline Accounting & Accounting Review & 12.1 & 20.9 & 12.3 & 32.4 \\
\hline Anthropology & Current Anthropology & 11.2 & 17.8 & 61.4 & 57.3 \\
\hline Anthropology & Amer. Jrn. of Physical Anthropology & 8.9 & 14.0 & 26.6 & 47.3 \\
\hline Biology & Cell & 9.1 & 11.5 & 35.4 & 49.0 \\
\hline Chemistry & Journal of the Amer. Chemical Society & 6.4 & 7.9 & 32.8 & 38.6 \\
\hline Communication & Journal of Communication & 9.0 & 18.6 & 10.0 & 52.7 \\
\hline Computer Science & IEEE Transactions on Info. Theory & 7.3 & 13.7 & 17.5 & 23.9 \\
\hline Computer Science & Journal of the ACM & 12.0 & 29.0 & 15.0 & 37.8 \\
\hline Computer Science & Communications of the ACM & 7.6 & 7.0 & 14.6 & 6.9 \\
\hline Demography & Demography & 13.7 & 13.5 & 16.9 & 37.5 \\
\hline Ecology & Ecology & 9.8 & 12.7 & 33.0 & 56.2 \\
\hline Economics & Econometrica & 14.8 & 31.1 & 15.1 & 27.2 \\
\hline Economics & Journal of Political Economy & 22.2 & 29.7 & 18.0 & 29.7 \\
\hline Economics & Quarterly Journal of Economics & 15.5 & 36.7 & 15.9 & 31.2 \\
\hline Economics & Review of Economic Studies & 12.9 & 23.5 & 12.2 & 29.2 \\
\hline Education & American Educational Research Journal & 15.0 & 36.4 & 19.4 & 50.7 \\
\hline Education & Harvard Educational Review & 23.8 & 26.8 & 47.2 & 43.7 \\
\hline Eng. - Aero. & AIAA Journal & 5.8 & 7.8 & 11.5 & 21.4 \\
\hline Eng. - Elec. & IEEE Transactions on Electron Devices & 6.6 & 7.2 & 13.9 & 17.7 \\
\hline Eng. - Materials & Journal of Materials Science & 7.7 & 7.8 & 18.0 & 21.6 \\
\hline Eng. - Mech. & Journal of Eng. Mechanics and ASCE & 15.3 & 8.5 & 14.9 & 19.2 \\
\hline Finance & Journal of Finance & 13.9 & 34.8 & 15.0 & 30.8 \\
\hline Geography & Ann. of the Assoc. of Amer. Geographers & 12.7 & 24.4 & 37.1 & 67.1 \\
\hline Geology & Geology & 4.8 & 4.0 & 19.8 & 25.2 \\
\hline Geology & Journal of Geology & 17.8 & 15.3 & 35.7 & 50.7 \\
\hline History & American Historical Review & 22.5 & 31.0 & 85.9 & 62.7 \\
\hline History & Journal of American History & 22.8 & 28.4 & 75.2 & 65.9 \\
\hline Law & Harvard Law Review & 59.1 & 92.4 & 156.6 & 47.7 \\
\hline Law & Yale Law Journal & 56.2 & 85.4 & 165.9 & 59.1 \\
\hline Linguistics & Language & 18.8 & ${ }^{a} 30.1$ & 30.9 & 61.2 \\
\hline Mathematics & Annals of Mathematics & 21.6 & 35.0 & 18.0 & 28.4 \\
\hline Mathematics & Inventiones Mathematicae & 22.7 & 34.6 & 16.1 & 25.2 \\
\hline Medicine & New England Journal of Medicine & 4.0 & 7.2 & 23.0 & 24.9 \\
\hline Medicine & Journal of the Amer. Medical Assoc. & 4.1 & 6.6 & 9.5 & 24.2 \\
\hline Oceanography & Limnology and Oceanography & 10.2 & 11.3 & 26.5 & 42.3 \\
\hline Paleontology & Journal of Paleontology & 13.8 & 12.8 & 19.2 & 45.4 \\
\hline Philosophy & Philosophy of Science & 15.8 & 20.6 & 16.0 & 32.3 \\
\hline Physics & Physical Review Letters & 3.3 & 4.0 & 15.4 & 20.8 \\
\hline Physics & Physical Review B & 8.9 & 8.5 & 25.4 & 28.6 \\
\hline Physics & Physical Review D & 8.9 & 10.7 & 22.9 & 31.5 \\
\hline Political Science & American Journal of Political Science & 16.9 & 24.2 & 27.6 & 37.2 \\
\hline Political Science & American Political Science Review & 12.7 & ${ }^{a} 16.1$ & 37.1 & 51.0 \\
\hline Psychology & Psychological Review & 21.6 & ${ }^{a} 30.3$ & 51.4 & 60.0 \\
\hline Psychology & Journal of Personality and Social Psych. & 8.3 & 14.4 & 23.8 & 59.3 \\
\hline Psychology & Developmental Psychology & 7.8 & 11.5 & 12.6 & 45.8 \\
\hline Sciences & Nature & 4.2 & 5.7 & 17.8 & 25.8 \\
\hline Sciences & Science & 7.7 & 5.5 & 23.3 & 28.5 \\
\hline Sociology & American Sociological Review & 15.5 & 17.2 & 35.8 & 49.2 \\
\hline Sociology & American Journal of Sociology & 22.7 & 39.3 & 26.2 & 60.1 \\
\hline Statistics & Journal of the Amer. Statistical Assoc. & 5.9 & 11.5 & 13.5 & 28.3 \\
\hline Women's Studies & Signs & 22.1 & 31.0 & 22.5 & 48.5 \\
\hline
\end{tabular}

Notes: $a$ - Data not adjusted for larger page size. 
Table 2: Duration of the review process at various journals: 1975 and 1999

\begin{tabular}{|llrr|}
\hline & & & \\
& & Mean & delay \\
\cline { 2 - 4 } Field & Journal & 1975 & 1999 \\
\hline & Mean submission-final resubmission time & & \\
\hline Biology & Cell & 1.2 & 2.2 \\
Computer Science & IEEE Transactions on Info. Theory & 6.9 & 13.6 \\
Computer Science & Journal of the ACM & 10.4 & 21.0 \\
Economics & Econometrica & 9.3 & 26.3 \\
Eng. - Aero. & AIAA Journal & 5.3 & 8.7 \\
Eng. - Elec. & IEEE Transactions on Electron Devices & 4.6 & 6.0 \\
Eng. - Elec. & Proceedings of the IEEE & 2.6 & 6.9 \\
Eng. - Materials & Journal of Materials Science & 1.5 & 5.2 \\
Finance & Journal of Finance & 6.5 & 18.6 \\
Mathematics & Annals of Mathematics & 2.0 & 5.5 \\
Mathematics & Inventiones Mathematicae & 1.3 & 10.8 \\
Philosophy & Philosophy of Science & 0.9 & 5.8 \\
Physics & Physical Review B & 0.4 & 1.3 \\
Political Science & American Journal of Political Science & 6.0 & 7.6 \\
Psychology & Psychological Review & 1.7 & 18.8 \\
Statistics & Annals of Statistics & 15.0 & 18.0 \\
Statistics & Journal of the Amer. Statistical Assoc. & 10.1 & 18.6 \\
Stat. - Bio. & Biometrika & 5.3 & 15.1 \\
Stat. - Psych. & Psychometrika & 8.1 & 16.8 \\
\hline & Mean submission-acceptance time & & \\
\hline Ecology & Ecology & 8.9 & 69.2 \\
Economics & Quarterly Journal of Economics & 8.5 & 13.0 \\
Economics & Review of Economic Studies & 12.1 & 28.8 \\
Geology & Geology & 2.2 & 4.6 \\
Oceanography & Limnology and Oceanography & 7.0 & $c 8.8$ \\
\hline & Mean submission-publication time & & \\
\hline Astronomy & Astrophysical Journal & 7.9 & 10.7 \\
Chemistry & Journal of the Amer. Chemical Society & 7.8 & 6.2 \\
Geology & Journal of Geology & 12.4 & 10.6 \\
Linguistics & Language & 12.8 & 24.8 \\
Philosophy & Philosophy of the Social Sciences & 13.8 & 22.1 \\
Physics & Physical Review Letters & 2.1 \\
Psychology & Developmental Psychology & 5.8 \\
Sciences & Nature & 4.3 \\
\hline \hline
\end{tabular}

Notes: $a$ - Data for 1979. $b$ - Does not include time for post-acceptance revisions (which occurred for about $40 \%$ of papers.) $c$ - Does not include time for post-acceptance revisions (which occurred for about $90 \%$ of papers.) 